\title{
Freidlin-Wentzell's Large Deviations for Stochastic Evolution Equations with Poisson Jumps
}

\author{
Huiyan Zhao ${ }^{1,2}$, Siyan $\mathrm{Xu}^{3}$ \\ ${ }^{1}$ School of Economics and Statistics, Guangzhou University, Guangzhou, China \\ ${ }^{2}$ School of Applied Mathematics, Beijing Normal University Zhuhai, Zhuhai, China \\ ${ }^{3}$ Faculty of Science, Ningbo University, Ningbo, China \\ Email:huiyan.zhao@msn.com,xusiyan@nbu.edu.cn
}

How to cite this paper: Zhao, H.Y. and Xu, S.Y. (2016) Freidlin-Wentzell's Large Deviations for Stochastic Evolution Equations with Poisson Jumps. Advances in Pure Mathematics, 6, 676-694.

http://dx.doi.org/10.4236/apm.2016.610056

Received: August 4, 2016

Accepted: September 16, 2016

Published: September 19, 2016

Copyright $\odot 2016$ by authors and Scientific Research Publishing Inc. This work is licensed under the Creative Commons Attribution International License (CC BY 4.0).

http://creativecommons.org/licenses/by/4.0/

(c) (i) Open Access

\begin{abstract}
We establish a Freidlin-Wentzell's large deviation principle for general stochastic evolution equations with Poisson jumps and small multiplicative noises by using weak convergence method.
\end{abstract}

\section{Keywords}

Stochastic Evolution Equation, Poisson Jumps, Freidlin-Wentzell's Large Deviation, Weak Convergence Method

\section{Introduction}

The weak convergence method of proving a large deviation principle has been developed by Dupuis and Ellis in [1]. The main idea is to get sevral variational representation formulas for the Laplace transform of certain functionals, and then to prove an equivalence between Laplace principle and large deviation principle (LDP). For Brownian functionals, Boué and Dupuis [2] have proved an elegant variational representation formula (also can be found in Zhang [3]). For Poisson functionals, we can see Zhang [4]. Recently, a variational representation formula on Wiener-Poisson space has been established by Budhiraja, Dupuis, and Maroulas in [5]. These type variational representations have been proved to be very effective for both finite-dimensional and infinite-dimensional stochastic dynamical systems (cf. [6]-[10]). The main advantages of this method are that we only have to make some necessary moment estimates.

However, there are still few results on the large deviation for stochastic evolution equations with jumps. In [11], Röckner and Zhang considered the following type 
semi-linear stochastic evolutions driven by Lévy processes

$$
\left\{\begin{array}{l}
\mathrm{d} Z_{t}^{\epsilon}=A Z_{t}^{\epsilon} \mathrm{d} t+b \mathrm{~d} t+\sqrt{\epsilon} \mathrm{d} W_{t}+\int_{\mathbb{X}} f(x)\left(\epsilon N^{\epsilon^{-1}}(\mathrm{~d} x, \mathrm{~d} t)-v(\mathrm{~d} x) \mathrm{d} t\right), \epsilon \in(0,1], \\
Z_{0}^{\epsilon}=z \in H,
\end{array}\right.
$$

they established the LDP by proving some exponential integrability on different spaces. Later, Budhiraja, Chen and Dupuis developed a large deviation for small Poisson perturbations of a more general class of deterministic equations in infinite dimensional ([12]), but they did not consider the small Brownian perturbations simultaneously.

Motivated by the above work, we would like to prove a Freidlin-Wentzell's large deviation for nonlinear stochastic evolution equations with Poisson jumps and Brownian motions. At the same time, nonlinear stochastic evolution equations have been studied in various literatures (cf. [13]-[17]). So we consider the following stochastic evolution equation:

$$
\left\{\begin{array}{l}
\mathrm{d} Z_{t}^{\epsilon}=A\left(t, Z_{t}^{\epsilon}\right) \mathrm{d} t+\sqrt{\epsilon} B\left(t, Z_{t}^{\epsilon}\right) \mathrm{d} W_{t}+\int_{\mathbb{X}} f\left(t, Z_{t-}^{\epsilon}, x\right)\left(\epsilon N^{\epsilon^{-1}}(\mathrm{~d} x, \mathrm{~d} t)-v(\mathrm{~d} x) \mathrm{d} t\right), \epsilon \in(0,1], \\
Z_{0}^{\epsilon}=Z \in H,
\end{array}\right.
$$

in the framework of a Gelfand's triple:

$$
V \subset H \equiv H^{*} \subset V^{*}
$$

where $V, H$ (see Section 2) are separable Banach and separable Hilbert space respectively. We will establish LDP for solutions of above evolution equation on $D([0, T] ; H) \cap L^{2}([0, T] ; V)$, where $D([0, T] ; H)$ is $H$-valued cádlág function space with the Skorokhod topology. For stochastic evolution equations without jumps, Ren and Zhang [9] and Liu [8] achieved the LDP on $C([0, T] ; H) \cap L^{q}([0, T] ; \mathrm{d} t)(q \geq 2)$ and $C([0, T] ; H) \cap L^{q}([0, T] ; \mathrm{d} t) \quad(q>1)$ respectively. In our case, there are two new difficulties. The first one is to find a sufficient condition to characterize a compact set in $D\left([0, T] ; V^{*}\right)$ (see Proposition 4) instead of Ascoli-Arzelà's theorem for continuous case, the second one is to control the jump parts. This form of equation contains a large class of (nonliear) stochastic partial differential equation of evolutional type, for applications and examples we refer the reader to [8], [9]. The equations we consider here are more general than the equations considered in [11], and we use a different method. We note that, the large deviations for semilinear SPDEs in the sense of mild solutions were considered in paper [18] recently. For other recent research on this topic, see also [12], [19].

In Section 2, we firstly give some notations and recall some results from [5], which are the basis of our paper, and then introduce our framework. In Section 3, we prove the large deviation principle. In the last section, we give an application. Note that notations $c, C_{M}$ and $C_{T, M}$ below will only denote positive constants whose values may vary from line to line.

\section{Preliminaries and Framework}

We first recall some notations from [5]. 
Let $\mathbb{X}$ be a locally compact Polish space and denote by $\mathcal{M}(\mathbb{X})$ the space of all measures $v$ on $(\mathbb{X}, \mathcal{B}(\mathbb{X}))$, satisfying $v(\Gamma)<\infty$ for every compact $\Gamma \subset \mathbb{X}$. Let $C_{c}(\mathbb{X})$ be the space of continuous functions with compact support. $\mathcal{M}(\mathbb{X})$ is a Polish space endowed with the weakest topology such that for every $f \in C_{c}(\mathbb{X})$, $\mathcal{M}(\mathbb{X}) \ni v \rightarrow, v:=\int_{\mathbb{X}} f(u) v(\mathrm{~d} u)$ is a continuous function.

Set $\mathbb{Y}:=\mathbb{X} \times[0, \infty)$. Fix $T \in(0, \infty)$ and let $\mathbb{Y}_{T}:=[0, T] \times \mathbb{Y}$. Let $\mathbb{M}:=\mathcal{M}\left(\mathbb{Y}_{T}\right)$ and denote by $P_{1}$ the unique probability measure on $(\mathbb{M}, \mathcal{B}(\mathbb{M}))$ such that the canonical map, $\quad \tilde{N}: \mathbb{N} \rightarrow \mathbb{M}, \quad \tilde{N}(m):=m$, is a Poisson random measure with intensity $v_{T}:=\lambda_{T} \otimes v \otimes \lambda_{\infty}$, where $v \in \mathcal{M}(\mathbb{X}), \lambda_{T}$ and $\lambda_{\infty}$ are Lebesgue measures on $[0, T]$ and $[0, \infty)$ respectively.

Let $G$ be a real separable Hilbert space and let $Q$ be a positive definite and symmetric trace operator defined on $G$. Set $\mathbb{W}:=C([0, T] ; G)$ and $\Omega:=\mathbb{W} \times \mathbb{M}$. Let $N: \Omega \rightarrow \mathbb{M}$ be defined by $N(w, m)=m$, for $(w, m) \in \Omega$. Let $W$ be the coordinate map on $\Omega$ defined as $W(w, m):=w$. Define $\mathcal{G}_{t}:=\sigma\left\{N((0, s] \times A), W_{s}: 0 \leq s \leq t, A \in \mathcal{B}(\mathbb{Y})\right\}$. We denote by $P$ the unique probability measure on $(\Omega, \mathcal{B}(\Omega))$ such that under $P$.

1) $\mathrm{W}$ is a $\mathrm{Q}-\mathrm{Wiener}$ process;

2) $\mathrm{N}$ is a Poisson random measure with intensity measure $v_{T}$;

3) $\left\{W_{t}, t \in[0, T]\right\},\{N([0, t], A), t \in[0, T]\}$ are $\mathcal{G}_{t}$-martingales for every $A \in \mathcal{B}(\mathbb{X})$. We denote by $\mathcal{F}_{t}$ be $P$-completion of the filtration $\mathcal{G}_{t}$. From now on, we will work on the probability space $(\Omega, \mathcal{B}(\Omega), P)$ with filtration $\left\{\mathcal{F}_{t}, 0 \leq t \leq T\right\}$.

Denote by $\mathcal{P}$ the predictable $\sigma$-field on $[0, T] \times \Omega$ with the filtration $\left\{\mathcal{F}_{t}: 0 \leq t \leq T\right\}$ on $(\Omega, \mathcal{B}(\Omega))$. Let $\mathcal{A}:=\{f:[0, T] \times \mathbb{X} \times \Omega \rightarrow[0, \infty), f$ is $\mathcal{P} \otimes \mathcal{B}(\mathbb{X}) \backslash \mathcal{B}([0, \infty))$ measurable $\} . \forall \varphi \in \mathcal{A}$, define

$$
L_{T}(\varphi):=\int_{[0, T] \times \mathbb{X}} l(\varphi(t, x, \omega)) \mathrm{d} t v(\mathrm{~d} x),
$$

where

$$
l(r):=r \log (r)-r+1, \quad r \in[0, \infty)
$$

and define a counting process $N^{\varphi}$ as

$$
N^{\varphi}((0, t] \times U):=\int_{(0, t] \times U} \int_{(0, \infty)} 1_{[0, \varphi(s, x)]}(r) N(\mathrm{~d} s, \mathrm{~d} x, \mathrm{~d} r), \quad t \in[0, T], U \in \mathcal{B}(\mathbb{X}) .
$$

For fixed $M \in \mathbb{N}$, let

$$
\tilde{S}_{M}:=\left\{g:[0, T] \times \mathbb{X} \rightarrow[0, \infty): L_{T}(g) \leq M\right\} .
$$

By [5], we can define $v_{T}^{g}(A):=\int_{A} g(s, x) v(\mathrm{~d} x) \mathrm{d} s, A \in \mathcal{B}([0, T] \times \mathbb{X})$ for a function $g \in \tilde{S}_{M}$, and identify $g$ with measure $v_{T}^{g}$. Besides, $\left\{v_{T}^{g}: g \in \tilde{S}_{M}\right\}$ is a compact subset of $\mathcal{M}([0, T] \times \mathbb{X})$ through the superlinear groth of 1 . We can also consider the topology on $\tilde{S}_{M}$ which makes $\tilde{S}_{M}$ a compact space.

Remark 1. We note that, for $g_{n}, g \in \tilde{S}_{M}, g_{n} \rightarrow g$ in this topology means $v_{T}^{g_{n}} \rightarrow v_{T}^{g}$, that is, for any $f \in C_{c}([0, T] \times \mathbb{X} ; \mathbb{R})$, $\left|\int_{0}^{T} \int_{\mathbb{X}} f(s, x)\left(g_{n}(s, x)-g(s, x)\right) v(\mathrm{~d} x) \mathrm{d} s\right| \rightarrow 0$ holds as $n \rightarrow \infty$. 
Set $G_{Q}:=Q^{1 / 2}(G)$ and define $\|v\|_{G_{Q}}:=\left\|\left(Q^{-1}\right)^{1 / 2} v\right\|_{G}$. Let

$$
\begin{gathered}
\mathcal{P}_{2}:=\left\{\psi: \mathcal{P} \backslash \mathcal{B}\left(G_{Q}\right) \text { measurable and } \int_{0}^{T}\|\psi(s)\|_{G_{Q}}^{2} \mathrm{~d} s<\infty \text { a.s. } P\right\} \\
\bar{S}_{M}:=\left\{h \in L^{2}\left([0, T] ; G_{Q}\right): \frac{1}{2} \int_{0}^{T}\|h(s)\|_{G_{Q}}^{2} \mathrm{~d} s \leq M\right\} .
\end{gathered}
$$

We endow $\bar{S}_{M}$ with the weak topology on the Hilbert space such that $\bar{S}_{M}$ is a compact subset of $L^{2}\left([0, T] ; G_{Q}\right)$.

Let $S_{M}:=\bar{S}_{M} \times \tilde{S}_{M}$ with the usual product topology. Set $\mathcal{U}:=\mathcal{P}_{2} \times \mathcal{A}$ and let $\mathcal{U}_{M}$ be the space of $S_{M}$-valued controls:

$$
\mathcal{U}_{M}:=\left\{u=(\psi, \varphi) \in \mathcal{U}: u(\omega) \in S_{M}, P \text { a.e. } \omega\right\} .
$$

Let $\mathbb{D}$ be a Polish space and let $\left\{X^{\epsilon}\right\}_{\epsilon>0}$ be a set of $\mathbb{D}$-valued random variables defined on $(\Omega, \mathcal{B}(\Omega), P)$ by

$$
X^{\epsilon}:=\mathcal{G}^{\epsilon}\left(\sqrt{\epsilon} W, \epsilon N^{\epsilon^{-1}}\right),
$$

where $\left\{\mathcal{G}^{\epsilon}\right\}_{\epsilon>0}$ is a family of measurable maps from $\Omega$ to $\mathbb{D}$.

Hypothesis. There exists a measurable map $\mathcal{G}^{0}: \Omega \rightarrow \mathbb{D}$ such that the following hold.

1) For $M \in \mathbb{N}$, if a family $\left\{u_{\epsilon}=\left(\psi_{\epsilon}, \varphi_{\epsilon}\right), \epsilon \in(0,1)\right\} \subset \mathcal{U}_{M}$ converges in distribution to $u=(\psi, \varphi) \in \mathcal{U}_{M}$, then

$$
\mathcal{G}^{\epsilon}\left(\sqrt{\epsilon} W+\int_{0} \psi_{\epsilon}(s) \mathrm{d} s, \epsilon N^{\epsilon^{-1} \varphi_{\epsilon}}\right) \Rightarrow \mathcal{G}^{0}\left(\int_{0} \psi(s) \mathrm{d} s, v_{T}^{\varphi}\right)
$$

where $\Rightarrow$ denotes the weak convergence.

2) For $M \in \mathbb{N}$, let $\left(h_{m}, g_{m}\right),(h, g) \in S_{M}$ be such that $\left(h_{m}, g_{m}\right) \rightarrow(h, g)$. Then

$$
\mathcal{G}^{0}\left(\int_{0} h_{m}(s) \mathrm{d} s, v_{T}^{g_{m}}\right) \rightarrow \mathcal{G}^{0}\left(\int_{0}^{\cdot} h(s) \mathrm{d} s, v_{T}^{g}\right) .
$$

For $\phi \in \mathbb{D}$, define $S_{\phi}:=\left\{(h, g) \in S: \phi=\mathcal{G}^{0}\left(\int_{0} h(s) \mathrm{d} s, v_{T}^{g}\right)\right\}$. Let $I: \mathbb{D} \rightarrow[0, \infty]$ be

$$
I(\phi)=\inf _{(h, g) \in S_{\phi}}\left\{\frac{1}{2} \int_{0}^{T}\|h(s)\|_{G_{Q}}^{2} \mathrm{~d} s+L_{T}(g)\right\}
$$

where $\inf \varnothing:=\infty$.

We have the following important result due to [5].

Theorem 2. Under the above Hypothesis, $\left\{X^{\epsilon}\right\}_{\epsilon>0}$ satisfies a large deviation principle with rate function $I$.

Now we introduce our framework and assumptions.

Let $\left(H,\langle\cdot, \cdot\rangle_{H}\right)$ be a real separable Hilbert space. Let $V$ be a reflexive Banach space and $V^{*}$ be the dual space of $V$ and ${ }_{V^{*}}\langle\cdot, \cdot\rangle_{V}$ denotes the corresponding dualization. Identify $H$ with its dual $H^{*}$ and the following assumptions are satisfied:

1) $V \subset H \equiv H^{*} \subset V^{*}$;

2) $V$ is dense in $H$;

3) there exists a constant $c$ such that for all $v \in V,\|v\|_{H} \leq c\|v\|_{V}$;

4) $\left.V^{*}\langle\cdot, \cdot\rangle_{V}\right|_{H \times V}=\langle\cdot, \cdot\rangle_{H}$. 
Let $L_{2}(G ; H)$ be the space of Hilbert-Schmidt linear operators from $G$ to $H$, which is a real separable Hilbert space with the inner product

$$
\left\langle B_{1}, B_{2}\right\rangle_{L_{2}(G ; H)}:=\sum_{i \geq 1}\left\langle B_{1} g_{i}, B_{2} g_{i}\right\rangle_{H},
$$

where $\left\{g_{i}\right\}$ is an orthonormal basis of $G$. We denote by $L_{Q}(G ; H)$ the set of all linear operators $C$ mapping $Q^{1 / 2} G$ into $H$ such that $C Q^{1 / 2} \in L_{2}(G ; H)$, and the norm $\|C\|_{L_{Q}}:=\left\|C Q^{1 / 2}\right\|_{L_{2}}$

Let

$$
\begin{gathered}
A:[0, T] \times V \times \Omega \rightarrow V^{*}, \\
B:[0, T] \times V \times \Omega \rightarrow L_{Q}(G ; H), \\
f:[0, T] \times V \times \mathbb{X} \times \Omega \rightarrow V
\end{gathered}
$$

be progressively measurable. For example, for every $t \in[0, T], A$ restricted to $[0, T] \times V \times \Omega$ is $\mathcal{B}([0, T]) \otimes \mathcal{B}(V) \otimes \mathcal{F}_{t}$-measurable

We assume throughout this paper that:

(H1) Hermicontinuity: For any $u, v, x \in V, \omega \in \Omega$ and any $t \in[0, T]$, the mapping

$$
[0,1] \ni \varepsilon \mapsto{ }_{V^{*}}\langle A(t, u+\varepsilon v, \omega), x\rangle_{V}
$$

is continuous.

(H2) Weak monotonicity: There exist $\lambda_{0}, \lambda_{1} \geq 0$ such that for all $u, v \in V$

$$
2_{V^{*}}\left\langle A(\cdot, u)-A(\cdot, v), u-v_{V}\right\rangle \leq-\lambda_{1}\|u-v\|_{V}^{2}+\lambda_{0}\|u-v\|_{H}^{2}
$$

holds on $[0, T] \times \Omega$.

(H3) Coercivity: For all $v \in V$ and $t \in[0, T]$, there exist $c_{1}, c_{2}>0$ such that

$$
2_{V^{*}}\langle A(t, v), v\rangle_{V} \leq c_{1}\|v\|_{H}^{2}-c_{2}\|v\|_{V}^{2}
$$

holds on $\Omega$.

(H4) For all $t \in[0, T]$ and $u \in V$, there exists $c_{3} \geq 0$ such that

$$
\|A(t, u)\|_{V^{*}} \leq c_{3}\left(1+\|u\|_{V}\right)
$$

holds on $\Omega$.

(H5) There exists $c_{4}>0$ such that for all $u, v \in V, \quad x \in \mathbb{X}$ and $t \in[0, T]$

$$
\begin{gathered}
\|B(t, u)-B(t, v)\|_{L_{Q}} \leq c_{4}\|u-v\|_{H}, \\
\|f(t, u, x)-f(t, v, x)\|_{H} \leq c_{4}\|u-v\|_{H}, \\
\|B(t, u)\|_{L_{Q}} \leq c_{4}\left(1+\|u\|_{H}\right),
\end{gathered}
$$

and

$$
\|f(t, u, x)\|_{H} \leq c_{4}\left(1+\|u\|_{V^{*}}\right) .
$$

(H6) There exist some compact $\Gamma \subset \mathbb{X}, f(t, u, x)=0$, for all $(t, u, x) \in[0, T] \times V \times \Gamma^{c}$. For any $u \in V, f(\cdot, u, \cdot)$ is continuous on $[0, T] \times \Gamma$. 
(H7) $V \hookrightarrow H$ compactly.

\section{Large Deviation Principle}

Consider small noise stochastic evolution equation as following:

$\left\{\begin{array}{l}\mathrm{d} Z_{t}^{\epsilon}=A\left(t, Z_{t}^{\epsilon}\right) \mathrm{d} t+\sqrt{\varepsilon} B\left(t, Z_{t}^{\epsilon}\right) \mathrm{d} W_{t}+\int_{\mathbb{X}} f\left(t, Z_{t-}^{\epsilon}, x\right)\left(\epsilon N^{\epsilon^{-1}}(\mathrm{~d} x, \mathrm{~d} t)-v(\mathrm{~d} x) \mathrm{d} t\right), \epsilon \in(0,1], \\ Z_{0}^{\epsilon}=Z \in H .\end{array}\right.$

Under the assumptions (H1)-(H5), by [15], [17], there exists a unique solution in $D([0, T] ; H) \cap L^{2}([0, T] ; V)$ to Equation (5). By Yamada-Watanabe theorem, there exists a measurable mapping $\mathcal{G}^{\epsilon}: \Omega \rightarrow D([0, T] ; H) \cap L^{2}([0, T] ; V)$ such that

$$
Z^{\epsilon}=\mathcal{G}^{\epsilon}\left(\sqrt{\epsilon} W, \epsilon N^{\epsilon^{-1}}\right) \text {. }
$$

We now fix a family of processes $\left(\psi_{\epsilon}, \varphi_{\epsilon}\right) \in \mathcal{U}_{M}$, and put

$$
\tilde{Z}^{\epsilon}=\mathcal{G}^{\epsilon}\left(\sqrt{\epsilon} W+\int_{0} \psi_{\epsilon}(s) \mathrm{d} s, \epsilon N^{\epsilon^{-1} \varphi_{\epsilon}}\right) .
$$

By Girsanov's theorem, $\tilde{Z}^{\epsilon}$ is the unique solution of the following controlled stochastic evolution equation:

$$
\left\{\begin{aligned}
\mathrm{d} \tilde{Z}_{t}^{\epsilon}= & \left(A\left(t, \tilde{Z}_{t}^{\epsilon}\right)+B\left(t, \tilde{Z}_{t}^{\epsilon}\right) \psi_{\epsilon}(t)\right) \mathrm{d} t+\sqrt{\epsilon} B\left(t, \tilde{Z}_{t}^{\epsilon}\right) \mathrm{d} W_{t} \\
& +\int_{\mathbb{X}} f\left(t, \tilde{Z}_{t-}^{\epsilon}, x\right)\left(\epsilon N^{\epsilon^{-1} \varphi_{\epsilon}}(\mathrm{d} x, \mathrm{~d} t)-v(\mathrm{~d} x) \mathrm{d} t\right), \epsilon \in(0,1], \\
\tilde{Z}_{0}^{\epsilon}= & z \in H
\end{aligned}\right.
$$

Remark 3. For $\left(\psi_{\epsilon}, \varphi_{\epsilon}\right) \in \mathcal{U}_{M}$, by (1) and (2), there exists a constant $C_{M}>0$ such that for all $\epsilon \in(0,1]$,

$$
\int_{0}^{T}\left\|\psi_{\epsilon}(s)\right\|_{G_{Q}}^{2} \mathrm{~d} s+\int_{0}^{T} \int_{\mathbb{X}} \phi_{\epsilon}(s, x) v(\mathrm{~d} x) \mathrm{d} s<C_{M} \quad \text { a.s.. }
$$

We will verify that $\mathcal{G}^{\epsilon}$ satisfies the Hypothesis with $\mathbb{D}$ replaced by $D([0, T] ; H) \cap L^{2}([0, T] ; V)$. By using the similar method as in [9], we have the following uniform estimates about $\tilde{Z}^{\epsilon}$.

Lemma 1. There exists a constant $c_{T, M}>0$ such that, for all $\epsilon \in(0,1]$,

$$
\begin{gathered}
\mathbb{E}\left(\sup _{t \in[0, T]}\left\|\tilde{Z}_{s}^{\epsilon}\right\|_{H}^{2}\right)+\mathbb{E} \int_{0}^{T}\left\|\tilde{Z}_{s}^{\epsilon}\right\|_{V}^{2} \mathrm{~d} s \leq c_{T, M}\left(\|z\|_{H}^{2}+1\right), \\
\mathbb{E}\left(\sup _{t \in[0, T]}\left\|\tilde{Z}_{s}^{\epsilon}\right\|_{H}^{4}\right) \leq c_{T, M}\left(\|z\|_{H}^{4}+1\right) .
\end{gathered}
$$

In order to characterize a compact set in $D\left([0, T] ; V^{*}\right)$, we need the following lemma.

Lemma 2. For any $\delta>0$ and $\eta>0$, there exist $\epsilon_{0}>0$ and $\theta>0$ such that for any $\epsilon \leq \epsilon_{0}$, we have

$$
P\left(\sup _{t, s \in[0, T], t-s \mid<\theta}\left\|\tilde{Z}_{t}^{\epsilon}-\tilde{Z}_{s}^{\epsilon}\right\|_{V^{*}}>\eta\right) \leq \delta
$$


Proof. For fixed $\theta>0$ and any $t$ such that $0 \leq t \leq t+\theta \leq T$, we have

$$
\begin{aligned}
\tilde{Z}_{t+\theta}^{\epsilon}-\tilde{Z}_{t}^{\epsilon}= & \int_{t}^{t+\theta}\left(A\left(s, \tilde{Z}_{s}^{\epsilon}\right)+B\left(s, \tilde{Z}_{s}^{\epsilon}\right) \psi_{\epsilon}(s)\right) \mathrm{d} s+\int_{t}^{t+\theta} \sqrt{\epsilon} B\left(s, \tilde{Z}_{s}^{\epsilon}\right) \mathrm{d} W_{s} \\
& +\int_{t}^{t+\theta} \int_{\mathbb{X}} f\left(s, \tilde{Z}_{s-}^{\epsilon}, x\right)\left(\epsilon N^{\epsilon^{-1} \varphi_{\epsilon}}(\mathrm{d} s, \mathrm{~d} x)-v(\mathrm{~d} x) \mathrm{d} s\right) . \\
= & \int_{t}^{t+\theta}\left(A\left(s, \tilde{Z}_{s}^{\epsilon}\right)+B\left(s, \tilde{Z}_{s}^{\epsilon}\right) \psi_{\epsilon}(s)+\int_{\mathbb{X}} f\left(s, \tilde{Z}_{s-}^{\epsilon}, x\right)\left(\varphi_{\epsilon}(s, x)-1\right) v(\mathrm{~d} x)\right) \mathrm{d} s \\
& +\int_{t}^{t+\theta} \sqrt{\epsilon} B\left(s, \tilde{Z}_{s}^{\epsilon}\right) \mathrm{d} W_{s}+\int_{t}^{t+\theta} \int_{\mathbb{X}} f\left(s, \tilde{Z}_{s-}^{\epsilon}, x\right)\left(\epsilon N^{\epsilon^{-1} \varphi_{\epsilon}}(\mathrm{d} s, \mathrm{~d} x)-\varphi_{\epsilon}(s, x) \mathrm{d} s v(\mathrm{~d} x)\right) .
\end{aligned}
$$

Therefore

$$
\mathbb{E}\left(\sup _{t: 0 \leq t \leq t+\theta \leq T}\left\|\tilde{Z}_{t+\theta}^{\epsilon}-\tilde{Z}_{t}^{\epsilon}\right\|_{V^{*}}^{2}\right) \leq 3\left(I_{1}+I_{2}+I_{3}\right),
$$

where

$$
\begin{gathered}
I_{1}:=\mathbb{E}\left(\sup _{t: 0 \leq t \leq t+\theta \leq T}\left\|\int_{t}^{t+\theta}\left(A\left(s, \tilde{Z}_{s}^{\epsilon}\right)+B\left(s, \tilde{Z}_{s}^{\epsilon}\right) \psi_{\epsilon}(s)+\int_{\mathbb{X}} f\left(s, \tilde{Z}_{s-}^{\epsilon}, x\right)\left(\varphi_{\epsilon}(s, x)-1\right) v(\mathrm{~d} x)\right) \mathrm{d} s\right\|_{V^{*}}^{2}\right), \\
I_{2}:=\mathbb{E}\left(\sup _{t: 0 \leq t \leq t+\theta \leq T}\left\|\int_{t}^{t+\theta} \sqrt{\epsilon} B\left(s, \tilde{Z}_{s}^{\epsilon}\right) \mathrm{d} W_{s}\right\|_{V^{*}}^{2}\right), \\
I_{3}:=\mathbb{E}\left(\sup _{t: 0 \leq t \leq t+\theta \leq T}\left\|\int_{t}^{t+\theta} \int_{\mathbb{X}} f\left(s, \tilde{Z}_{s-}^{\epsilon}, x\right)\left(\epsilon N^{\epsilon^{-1} \varphi_{\epsilon}}(\mathrm{d} s, \mathrm{~d} x)-\varphi_{\epsilon}(s, x) \mathrm{d} s v(\mathrm{~d} x)\right)\right\|_{V^{*}}^{2}\right) .
\end{gathered}
$$

For $I_{1}$, by (H4), Hölder's inequality and Lemma 1, we have

$$
\begin{aligned}
I_{1} \leq & c \mathbb{E}\left(\sup _{t: 0 \leq t \leq t+\theta \leq T} \int_{t}^{t+\theta}\left\|A\left(s, \tilde{Z}_{s}^{\epsilon}\right)\right\|_{V^{*}} \mathrm{~d} s\right)^{2}+c \mathbb{E}\left(\sup _{t: 0 \leq t \leq t+\theta \leq T}\left\|\int_{t}^{t+\theta} B\left(s, \tilde{Z}_{s}^{\epsilon}\right) \psi_{\epsilon}(s) \mathrm{d} s\right\|_{H}^{2}\right) \\
& +c \mathbb{E}\left(\sup _{t: 0 \leq t \leq t+\theta \leq T}\left\|\int_{t}^{t+\theta} \int_{\mathbb{X}} f\left(s, \tilde{Z}_{s}^{\epsilon}, x\right)\left(\varphi_{\epsilon}(s, x)-1\right) v(\mathrm{~d} x) \mathrm{d} s\right\|_{H}^{2}\right) \\
\leq & c \mathbb{E}\left(\sup _{t: 0 \leq t \leq t+\theta \leq T} \int_{t}^{t+\theta}\left(1+\left\|\tilde{Z}_{s}^{\epsilon}\right\|_{V}\right) \mathrm{d} s\right)^{2}+c \mathbb{E}\left(\sup _{t: 0 \leq t \leq t+\theta \leq T} \int_{t}^{t+\theta}\left\|B\left(s, \tilde{Z}_{s}^{\epsilon}\right)\right\|_{L_{Q}}^{2} \mathrm{~d} s \int_{0}^{T}\left\|\psi_{\epsilon}(s)\right\|_{U_{Q}}^{2} \mathrm{~d} s\right) \\
& +c v(\Gamma) \mathbb{E}\left(\sup _{t: 0 \leq t \leq t+\theta \leq T} \int_{t}^{t+\theta} \int_{\Gamma}\left\|f\left(s, \tilde{Z}_{s}^{\epsilon}, x\right)\right\|_{H}^{2} v(\mathrm{~d} x) \mathrm{d} s\right) \\
& +c \mathbb{E}\left(\sup _{t: 0 \leq t \leq t+\theta \leq T} \int_{t}^{t+\theta} \int_{\mathbb{X}}\left\|f\left(s, \tilde{Z}_{s}^{\epsilon}, x\right)\right\|_{H}^{2} \varphi_{\epsilon}(s, x) v(\mathrm{~d} x) \mathrm{d} s \int_{t}^{t+\theta} \int_{\mathbb{X}} \varphi_{\epsilon}(s, x) v(\mathrm{~d} x) \mathrm{d} s\right) \\
\leq & c \mathbb{E}\left(2 \theta^{2}+2 \theta \int_{0}^{T}\left\|\tilde{Z}_{s}^{\epsilon}\right\|_{V}^{2} \mathrm{~d} s\right)+c \theta \mathbb{E}\left(1+\sup _{s \in[0, T]}\left\|\tilde{Z}_{s}^{\epsilon}\right\|_{H}^{2}\right)+c v(\Gamma)^{2} \theta^{2} \mathbb{E}\left(1+\sup _{s \in[0, T]}\left\|\tilde{Z}_{s}^{\epsilon}\right\|_{H}^{2}\right) \\
& +c \mathbb{E}\left(\left(1+\sup _{s \in[0, T]}\left\|\tilde{Z}_{s}^{\epsilon}\right\|_{H}^{2}\right) \sup _{t: 0 \leq t \leq t+\theta \leq T} \int_{t}^{t+\theta} \int_{\mathbb{X}} \varphi_{\epsilon}(s, x) v(\mathrm{~d} x) \mathrm{d} s\right) \\
\leq & c \theta^{2}+c \theta+c J_{\theta},
\end{aligned}
$$

where

$$
J_{\theta}:=\mathbb{E}\left(\left(1+\sup _{s \in[0, T]}\left\|\tilde{Z}_{s}^{\epsilon}\right\|_{H}^{2}\right) \sup _{t: 0 \leq t \leq t+\theta \leq T} \int_{t}^{t+\theta} \int_{\mathbb{X}} \varphi_{\epsilon}(s, x) v(\mathrm{~d} x) \mathrm{d} s\right) .
$$

By (7), we have

$$
\sup _{\epsilon \in(0,1] t: 0 \leq t \leq t+\theta \leq T} \sup _{t} \int_{t}^{t+\theta} \int_{\mathbb{X}} \varphi_{\epsilon}(s, x) v(\mathrm{~d} x) \mathrm{d} s \rightarrow 0, \text { a.s., } \quad \text { as } \theta \downarrow 0 .
$$


So by (9) and dominated convergence theorem, for all $\epsilon \in(0,1]$, we obtain

$$
J_{\theta} \rightarrow 0, \quad \text { as } \theta \downarrow 0 .
$$

For $I_{2}, I_{3}$, by BDG's inequality, (H5) and Lemma 1, we obtain

$$
I_{2} \leq 2 \epsilon \mathbb{E} \int_{0}^{T}\left\|B\left(s, \tilde{Z}_{s}^{\epsilon}\right)\right\|_{L_{Q}}^{2} \mathrm{~d} s \leq c \cdot \epsilon \mathbb{E} \int_{0}^{T}\left(1+\left\|\tilde{Z}_{s}^{\epsilon}\right\|_{H}^{2}\right) \mathrm{d} s \leq c \epsilon
$$

and

$$
\begin{aligned}
I_{3} & \leq 2 \mathbb{E}\left(\left\|\int_{0}^{T} \int_{\mathbb{X}} f\left(s, \tilde{Z}_{s-}^{\epsilon}, x\right)\left(\epsilon N^{\epsilon^{-1} \varphi_{\epsilon}}(\mathrm{d} s, \mathrm{~d} x)-\varphi_{\epsilon}(s, x) \mathrm{d} s v(\mathrm{~d} x)\right)\right\|_{V^{*}}^{2}\right) \\
& \leq \epsilon \mathbb{E} \int_{0}^{T} \int_{\mathbb{X}}\left\|f\left(s, \tilde{Z}_{s-}^{\epsilon}, x\right)\right\|^{2} \varphi_{\epsilon}(s, x) \mathrm{d} s v(\mathrm{~d} x) \\
& \leq \epsilon \mathbb{E} \int_{0}^{T} \int_{\mathbb{X}}\left(1+\left\|\tilde{Z}_{s}^{\epsilon}\right\|_{H}^{2}\right) \varphi_{\epsilon}(s, x) \mathrm{d} s v(\mathrm{~d} x) \\
& \leq \epsilon \mathbb{E} \int_{0}^{T} \int_{\mathbb{X}}\left(1+\left\|\tilde{Z}_{s}^{\epsilon}\right\|_{H}^{2}\right) \varphi_{\epsilon}(s, x) \mathrm{d} s v(\mathrm{~d} x)+\epsilon \mathbb{E}\left(\sup _{s \in[0, T]}\left\|\tilde{Z}_{s}^{\epsilon}\right\|_{H}^{2} \int_{0}^{T} \int_{\mathbb{X}} \varphi_{\epsilon}(s, x) \mathrm{d} s v(\mathrm{~d} x)\right) \\
& \leq c \epsilon .
\end{aligned}
$$

Hence, for any $\eta, \delta>0$

$$
\begin{aligned}
P\left(\sup _{t: 0 \leq t \leq t+\theta \leq T}\left\|\tilde{Z}_{t+\theta}^{\epsilon}-\tilde{Z}_{t}^{\epsilon}\right\|_{V^{*}}>\eta\right) & \leq P\left(\sup _{t: 0 \leq t \leq t+\theta \leq T}\left\|\tilde{Z}_{t+\theta}^{\epsilon}-\tilde{Z}_{t}^{\epsilon}\right\|_{V^{*}}^{2}>\eta^{2}\right) \\
& \leq \frac{3}{\eta^{2}}\left(I_{1}+I_{2}+I_{3}\right) \\
& \leq \frac{3}{\eta^{2}}\left(c \theta^{2}+c \theta+c J_{\theta}+2 c \epsilon\right),
\end{aligned}
$$

By choosing $\theta$ and $\epsilon$ small enough, then (10) holds immediately.

Proposition 4. For a sequence of $D\left([0, T] ; V^{*}\right)$-valued random variable $\left\{X^{n}\right\}$, if $\left\{X^{n}\right\}$ satisfies the following two conditons.

1) For any $\delta>0$, there are $n_{0} \in \mathbb{N}, R \in \mathbb{R}_{+}$, with

$$
n \geq n_{0} \Rightarrow P\left(\sup _{t \in[0, T]}\left\|X_{t}^{n}\right\|_{H}>R\right) \leq \delta
$$

2) For any $\delta>0$ and $\eta>0$, there are $n_{0} \in \mathbb{N}, \theta>0$, with

$$
n \geq n_{0} \Rightarrow P\left(\sup _{t, s \in[0, T], t-s \mid<\theta}\left\|X_{t}^{n}-X_{s}^{n}\right\|_{V^{*}}>\eta\right) \leq \delta
$$

Then $\left\{X^{n}\right\}$ is C-tight, that is, $\left\{X^{n}\right\}$ is tightness in $D\left([0, T] ; V^{*}\right)$ and if $X$ is a limit point then $X \in C\left([0, T] ; V^{*}\right)$ a.s..

Proof. It's obvious that (2) implies the following condition (cf. [20], p. 290). For any $\delta>0$ and $\eta>0$, there are $n_{0} \in \mathbb{N}, \theta_{0}>0$, with

$$
n \geq n_{0} \Rightarrow P\left(w\left(X^{n} ; \theta_{0}\right)>\eta\right) \leq \delta,
$$

where

$$
w\left(X^{n} ; \theta_{0}\right):=\inf \left\{\max _{i \leq r} \sup _{t, s \in\left[t_{i-1}, t_{i}\right)}\left\|X_{t}^{n}-X_{s}^{n}\right\|_{V^{*}}: 0=t_{0}<\cdots<t_{r}=T \inf _{i \leq r}\left(t_{i}-t_{i-1}\right) \geq \theta_{0}\right\} .
$$


For the finite family $\left(X^{n}\right)_{1 \leq n \leq n_{0}}$, we can find $R^{\prime}<\infty$ and $\theta^{\prime}>0$ such that

$$
\sup _{1 \leq n \leq n_{0}} P\left(\sup _{t \in[0, T]}\left\|X_{t}^{n}\right\|_{H}>R^{\prime}\right) \leq \delta, \quad \sup _{1 \leq n \leq n_{0}} P\left(w\left(X^{n} ; \theta^{\prime}\right)>\eta\right) \leq \delta .
$$

Hence, replacing $R$ by $R \vee R^{\prime}$ in (1) and $\theta_{0}$ by $\theta_{0} \wedge \theta^{\prime}$ in (11), we obtain that they still hold with $n_{0}=1$.

Fix $\delta>0$. Let $R_{\delta}<\infty$ and $\theta_{k, \delta}>0$ satisfy

$$
\sup _{n} P\left(\sup _{t \in[0, T]}\left\|X_{t}^{n}\right\|_{H}>R_{\delta}\right) \leq \frac{\delta}{2}, \sup _{n} P\left(w\left(X^{n} ; \theta_{k, \delta}\right)>\frac{1}{k}\right) \leq \frac{\delta}{2^{k+1}} .
$$

Then

$$
K_{\delta}:=\bigcap_{k=1}^{\infty}\left\{X \in D\left([0, T] ; V^{*}\right): \sup _{t \in[0, T]}\left\|X_{t}\right\|_{H} \leq R_{\delta}, w\left(X ; \theta_{k, \delta}\right) \leq \frac{1}{k}\right\}
$$

satisfies

$$
\sup _{n} P\left(X^{n} \notin K_{\delta}\right) \leq \sup _{n} P\left(\sup _{t \in[0, T]}\left\|X_{t}^{n}\right\|_{H}>R_{\delta}\right)+\sum_{k=1}^{\infty} \sup _{n} P\left(w\left(X^{n} ; \theta_{k, \delta}\right)>\frac{1}{k}\right) \leq \delta .
$$

By (H7), we have $H \equiv H^{*} \hookrightarrow V^{*}$ compactly. So, $K_{\delta}$ satisfies the conditions of Theorem A2.2 ([21], p. 563), then it's relatively compact in $D\left([0, T] ; V^{*}\right)$. This implies tightness of $\left\{X^{n}\right\}$.

It remains to prove that if a subsequence, still denoted by $\left(X^{n}\right)$, converges in law to some $X$, then $X$ is a.s. continuous. By taking the same scheme as in Proposition 3.26 (cf. [20], p. 315) and replacing $R^{d}$ by $V^{*}$ in the proof, we complete the proof.

According to Lemma 1 and Lemma 2, we have the following result:

Corollary 1. The sequence $\left\{\tilde{Z}^{\epsilon}\right\}_{\epsilon \in(0,1]}$ is C-tight in $D\left([0, T] ; V^{*}\right)$.

Lemma 3. Assume that for almost all $\omega,\left\{u_{\epsilon}=\left(\psi_{\epsilon}, \varphi_{\epsilon}\right)\right\}_{\epsilon \in(0,1]}$ weakly converges to $\{u=(\psi, \varphi)\}$ in $\mathcal{U}_{M}$ for fixed $M \in \mathbb{N}$ and there is a $C\left([0, T] ; V^{*}\right)$-valued process Z such that

$$
\sup _{0 \leq t \leq T}\left\|\tilde{Z}_{t}^{\epsilon}-\tilde{Z}\right\|_{t} \|_{V^{*}} \rightarrow 0 \quad \text { a.s.. }
$$

Then, $\tilde{Z}$ solves the following equation:

$$
\tilde{Z}_{t}=z+\int_{0}^{t} A\left(s, \tilde{Z}_{s}\right) \mathrm{d} s+\int_{0}^{t} B\left(s, \tilde{Z}_{s}\right) \psi(s) \mathrm{d} s+\int_{0}^{t} \int_{\mathbb{X}} f\left(s, \tilde{Z}_{s}, x\right)(\varphi(s, x)-1) v(\mathrm{~d} x) \mathrm{d} s .
$$

Moreover, we have

$$
\mathbb{E}\left(\sup _{0 \leq t \leq T}\left\|\tilde{Z}_{t}^{\epsilon}-\tilde{Z}_{t}\right\|_{H}^{2}\right) \rightarrow 0
$$

and if $\lambda_{1}>0$ in (H2), then

$$
\mathbb{E} \int_{0}^{T}\left\|\tilde{Z}_{t}^{\epsilon}-\tilde{Z}_{t}\right\|_{V}^{2} \mathrm{~d} t \rightarrow 0
$$

Proof. We divide our proof into several steps.

Step 1. By Lemma 1, we have 


$$
\sup _{\epsilon \in(0,1]} \mathbb{E}\left(\left\|\tilde{Z}_{t}^{\epsilon}\right\|_{H}^{2}\right)+\sup _{\epsilon \in(0,1]} \mathbb{E} \int_{0}^{T}\left\|\tilde{Z}_{t}^{\epsilon}\right\|_{V}^{2} \mathrm{~d} s<\infty
$$

and

$$
\sup _{\epsilon \in(0,1]} \mathbb{E}\left(\sup _{t \in[0, T]}\left\|\tilde{Z}_{t}^{\epsilon}\right\|_{H}^{4}\right)<\infty \text {. }
$$

Therefore, by the strong convergence of $\tilde{Z}^{\epsilon}(\cdot, \omega)$ to $\tilde{Z}(\cdot, \omega)$ as in (12). We get, for almost all $\omega, \tilde{Z}^{\epsilon}(T, \omega)$ converges weakly to $\tilde{Z}(T, \omega)$ in $H$ and $\tilde{Z}^{\epsilon}(\cdot, \omega)$ converges to $\tilde{Z}(\cdot, \omega)$ weakly in $L^{2}([0, T] ; V)$; and so we have

$$
\begin{aligned}
& \mathbb{E}\left(\|\tilde{Z}(T)\|_{H}^{2}\right) \leq \liminf _{\epsilon \downarrow 0} \mathbb{E}\left(\left\|\tilde{Z}_{T}^{\epsilon}\right\|_{H}^{2}\right)<\infty, \\
& \mathbb{E} \int_{0}^{T}\left\|\tilde{Z}_{s}\right\|_{V}^{2} \mathrm{~d} s \leq \liminf _{\epsilon \downarrow 0} \mathbb{E} \int_{0}^{T}\left\|\tilde{Z}_{s}^{\epsilon}\right\|_{V}^{2} \mathrm{~d} s<\infty .
\end{aligned}
$$

By (12), (16) and dominated convergence theorem, we have

$$
\lim _{\epsilon \downarrow 0} \mathbb{E}\left(\sup _{t \in[0, T]}\left\|\tilde{Z}_{t}^{\epsilon}-\tilde{Z}_{t}\right\|_{V^{*}}^{2}\right)=0 \text {. }
$$

Thus

$$
\begin{aligned}
\mathbb{E}\left(\int_{0}^{T}\left\|\tilde{Z}_{t}^{\epsilon}-\tilde{Z}_{t}\right\|_{H}^{2} \mathrm{~d} t\right) & =\mathbb{E}\left(\int_{0}^{T} V^{*}\left\langle\tilde{Z}_{t}^{\epsilon}-\tilde{Z}_{t}, \tilde{Z}_{t}^{\epsilon}-\tilde{Z}_{t}\right\rangle_{V} \mathrm{~d} t\right) \\
& \leq \mathbb{E}\left(\int_{0}^{T}\left\|\tilde{Z}_{t}^{\epsilon}-\tilde{Z}_{t}\right\|_{V^{*}}\left\|\tilde{Z}_{t}^{\epsilon}-\tilde{Z}_{t}\right\|_{V} \mathrm{~d} t\right) \\
& \leq\left(\int_{0}^{T} \mathbb{E}\left\|\tilde{Z}_{t}^{\epsilon}-\tilde{Z}_{t}\right\|_{V^{*}}^{2} \mathrm{~d} t \int_{0}^{T} \mathbb{E}\left\|\tilde{Z}_{t}^{\epsilon}-\tilde{Z}_{t}\right\|_{V}^{2} \mathrm{~d} t\right)^{1 / 2} \\
& \leq T^{1 / 2}\left(\mathbb{E}\left(\sup _{t \in[0, T]}\left\|\tilde{Z}_{t}^{\epsilon}-\tilde{Z}_{t}\right\|_{V^{*}}^{2}\right)\right)^{1 / 2} \mathbb{E}\left(\int_{0}^{T}\left\|\tilde{Z}_{t}^{\epsilon}-\tilde{Z}_{t}\right\|_{V}^{2} \mathrm{~d} t\right)^{1 / 2} \rightarrow 0, \text { as } \epsilon \downarrow 0 .
\end{aligned}
$$

Step 2. In this step, we prove $\tilde{Z}$ solves Equation (13). By (H4) and (15), we have

$$
\sup _{\epsilon \in(0,1]} \mathbb{E}\left(\int_{0}^{T}\left\|A\left(t, \tilde{Z}^{\epsilon}(t)\right)\right\|_{V^{*}}^{2} \mathrm{~d} t\right)<\infty .
$$

Hence, by (15) and (20), there exist subsequences of $\tilde{Z}^{\epsilon}, \tilde{Z}^{\epsilon}(T)$ and $A\left(\cdot, \tilde{Z}^{\epsilon}(\cdot)\right)$ (still denoted by themselves for simplicity) and $\bar{Z} \in L^{2}(\Omega \times[0, T] ; V), \quad \tilde{Z}_{T}^{\prime} \in L^{2}(\Omega ; H)$ and $Y \in L^{2}\left(\Omega \times[0, T] ; V^{*}\right)$ such that

$$
\begin{gathered}
\tilde{Z}^{\epsilon} \rightarrow \bar{Z} \quad \text { weakly in } L^{2}(\Omega \times[0, T] ; V), \\
\tilde{Z}^{\epsilon}(T) \rightarrow \tilde{Z}_{T}^{\prime} \quad \text { weakly in } L^{2}(\Omega ; H),
\end{gathered}
$$

and

$$
Y^{\epsilon}:=A\left(\cdot, \tilde{Z}^{\epsilon}(\cdot)\right) \rightarrow Y \quad \text { weakly in } L^{2}\left(\Omega \times[0, T] ; V^{*}\right)
$$

Define

$$
\hat{Z}_{t}:=z+\int_{0}^{t} Y_{s} \mathrm{~d} s+\int_{0}^{t} B\left(s, \tilde{Z}_{s}\right) \psi(s) \mathrm{d} s+\int_{0}^{t} \int_{\mathbb{X}} f\left(s, \tilde{Z}_{s}, x\right)(\varphi(s, x)-1) v(\mathrm{~d} x) \mathrm{d} s .
$$

Note that 


$$
\begin{aligned}
\tilde{Z}_{t}^{\epsilon}= & z+\int_{0}^{t} A\left(s, \tilde{Z}_{s}^{\epsilon}\right) \mathrm{d} s+\int_{0}^{t} B\left(s, \tilde{Z}_{s}^{\epsilon}\right) \psi_{\epsilon}(s) \mathrm{d} s+\sqrt{\epsilon} \int_{0}^{t} B\left(s, \tilde{Z}_{s}^{\epsilon}\right) \mathrm{d} W_{s} \\
& +\int_{0}^{t} \int_{\mathbb{X}} f\left(s, \tilde{Z}_{s-}^{\epsilon}, x\right)\left(\varphi_{\epsilon}(s, x)-1\right) v(\mathrm{~d} x) \mathrm{d} s \\
& +\epsilon \int_{0}^{t} \int_{\mathbb{X}} f\left(s, \tilde{Z}_{s-}^{\epsilon}, x\right)\left(N^{\epsilon^{-1} \varphi_{\epsilon}}(\mathrm{d} s, \mathrm{~d} x)-\epsilon^{-1} \varphi_{\epsilon}(s, x) v(\mathrm{~d} x) \mathrm{d} s\right) .
\end{aligned}
$$

By taking weak limits and by (19), we can get

$$
\hat{Z}(t, \omega)=\bar{Z}(t, \omega)=\tilde{Z}(t, \omega) \text { for } \mathrm{d} t \times \mathrm{d} P \text {-almost all }(t, \omega) .
$$

Indeed, for any $V$-valued bounded and measurable process $\xi$,

$$
\begin{aligned}
& \mathbb{E}\left(\int_{0}^{T}\left\langle\xi_{t}, \tilde{Z}_{t}^{\epsilon}-z\right\rangle_{H} \mathrm{~d} t\right)=\mathbb{E}\left(\int_{0}^{T} \int_{0}^{t}\left\langle A\left(s, \tilde{Z}_{s}^{\epsilon}\right), \xi_{t}\right\rangle_{V} \mathrm{~d} s \mathrm{~d} t\right) \\
& +\mathbb{E}\left(\int_{0}^{T}\left\langle\xi_{t}, \int_{0}^{t} B\left(s, \tilde{Z}_{s}^{\epsilon}\right) \psi_{\epsilon}(s) \mathrm{d} s\right\rangle_{H} \mathrm{~d} t\right)+\sqrt{\epsilon} \mathbb{E}\left(\int_{0}^{T}\left\langle\xi_{t}, \int_{0}^{t} B\left(s, \tilde{Z}_{s}^{\epsilon}\right) \mathrm{d} W_{s}\right\rangle_{H} \mathrm{~d} t\right) \\
& +\mathbb{E}\left(\int_{0}^{T}\left\langle\xi_{t}, \int_{0}^{t} \int_{\mathbb{X}} f\left(s, \tilde{Z}_{s-}^{\epsilon}, x\right)\left(\varphi_{\epsilon}(s, x)-1\right) v(\mathrm{~d} x) \mathrm{d} s\right\rangle_{H} \mathrm{~d} t\right) \\
& +\epsilon \mathbb{E}\left(\int_{0}^{T}\left\langle\xi_{t}, \int_{0}^{t} \int_{\mathbb{X}} f\left(s, \tilde{Z}_{s-}^{\epsilon}, x\right)\left(N^{\epsilon^{-1} \varphi_{\epsilon}}(\mathrm{d} s, \mathrm{~d} x)-\epsilon^{-1} \varphi_{\epsilon}(s, x) v(\mathrm{~d} x) \mathrm{d} s\right)\right\rangle_{H} \mathrm{~d} t\right) .
\end{aligned}
$$

By (21), (23) and taking limits for $\epsilon \downarrow 0$, then we get (see also the proof of (27) and (29) below)

$$
\mathbb{E}\left(\int_{0}^{T}\left\langle\xi_{t}, \bar{Z}_{t}-\hat{Z}_{t}\right\rangle_{H} \mathrm{~d} t\right)=0,
$$

which implies $\bar{Z}(t, \omega)=\hat{Z}(t, \omega)$ for almost all $(t, \omega)$. Similarly, we have $\hat{Z}_{T}(\omega)=\tilde{Z}_{T}^{\prime}(\omega)=\tilde{Z}_{T}(\omega)$ for almost all $\omega$.

We only have to prove

$$
Y(s, \omega)=A(s, \tilde{Z}(s, \omega)) \quad \text { for } \mathrm{d} t \times \mathrm{d} P \text {-almos tall }(t, \omega) .
$$

Let $\Phi \in L^{2}([0, T] ; V)$. By Itô's formula

$$
\begin{aligned}
\mathbb{E}\left(\mathrm{e}^{-2 \lambda_{0} T}\left\|\tilde{Z}_{T}^{\epsilon}\right\|_{H}^{2}\right)= & \|z\|_{H}^{2}-2 \lambda_{0} \mathbb{E}\left(\int_{0}^{T} \mathrm{e}^{-2 \lambda_{0} s}\left\|\tilde{Z}_{s}^{\epsilon}\right\|_{H}^{2} \mathrm{~d} s\right)+2 \mathbb{E}\left(\int_{0}^{T} \mathrm{e}^{-2 \lambda_{0} s} V^{*}\left\langle A\left(s, \tilde{Z}_{s}^{\epsilon}\right), \tilde{Z}_{s}^{\epsilon}\right\rangle_{V} \mathrm{~d} s\right) \\
& +2 \mathbb{E}\left(\int_{0}^{T} \mathrm{e}^{-2 \lambda_{0} s}\left\langle B\left(s, \tilde{Z}_{s}^{\epsilon}\right) \psi_{\epsilon}(s), \tilde{Z}_{s}^{\epsilon}\right\rangle_{H} \mathrm{~d} s\right)+\epsilon \mathbb{E}\left(\int_{0}^{T} \mathrm{e}^{-2 \lambda_{0} s}\left\|B\left(s, \tilde{Z}_{s}^{\epsilon}\right)\right\|_{L_{Q}}^{2} \mathrm{~d} s\right) \\
& +2 \mathbb{E}\left(\int_{0}^{T} \int_{\mathbb{X}} \mathrm{e}^{-2 \lambda_{0} s}\left\langle f\left(s, \tilde{Z}_{s-}^{\epsilon}, x\right), \tilde{Z}_{s}^{\epsilon}\right\rangle_{H}\left(\varphi_{\epsilon}(s, x)-1\right) v(\mathrm{~d} x) \mathrm{d} s\right) \\
& +\epsilon \mathbb{E}\left(\int_{0}^{T} \int_{\mathbb{X}} \mathrm{e}^{-2 \lambda_{0} s}\left\|f\left(s, \tilde{Z}_{s}^{\epsilon}, x\right)\right\|_{H}^{2} \varphi_{\epsilon}(s, x) v(\mathrm{~d} x) \mathrm{d} s\right) .
\end{aligned}
$$

By (H2)

$$
\begin{aligned}
& \left.2 \mathbb{E} \int_{0}^{T} \mathrm{e}^{-2 \lambda_{0} s}{ }_{V^{*}}\left\langle A\left(s, \tilde{Z}_{s}^{\epsilon}\right), \tilde{Z}_{s}^{\epsilon}\right\rangle_{V}-\lambda_{0}\left\|\tilde{Z}_{s}^{\epsilon}\right\|_{H}^{2}\right) \mathrm{d} s \\
& \leq 2 \mathbb{E}\left(\int_{0}^{T} \mathrm{e}^{-2 \lambda_{0} s} V^{*}\left\langle A\left(s, \tilde{Z}_{s}^{\epsilon}\right)-A\left(s, \Phi_{s}\right), \Phi_{s}\right\rangle_{V} \mathrm{~d} s\right)+2 \mathbb{E}\left(\int_{0}^{T} \mathrm{e}^{-2 \lambda_{0} s} V_{V^{*}}\left\langle A\left(s, \Phi_{s}\right), \tilde{Z}_{s}^{\epsilon}\right\rangle_{V} \mathrm{~d} s\right) \\
& \quad+2 \lambda_{0} \mathbb{E}\left(\int_{0}^{T} \mathrm{e}^{-2 \lambda_{0} s}\left(\left\|\Phi_{s}\right\|_{H}^{2}-2\left\langle\tilde{Z}_{s}^{\epsilon}, \Phi_{s}\right\rangle_{H}\right) \mathrm{d} s\right) \\
& \rightarrow 2 \mathbb{E}\left(\int_{0}^{T} \mathrm{e}^{-2 \lambda_{0} s} V^{*}\left\langle Y_{s}-A\left(s, \Phi_{s}\right), \Phi_{s}\right\rangle_{V} \mathrm{~d} s\right)+2 \mathbb{E}\left(\int_{0}^{T} \mathrm{e}^{-2 \lambda_{0} s} V^{*}\left\langle A\left(s, \Phi_{s}\right), \tilde{Z}_{s}\right\rangle_{V} \mathrm{~d} s\right) \\
& \quad+2 \lambda_{0} \mathbb{E}\left(\int_{0}^{T} \mathrm{e}^{-2 \lambda_{0} s}\left(\left\|\Phi_{s}\right\|_{H}^{2}-2\left\langle\tilde{Z}_{s}, \Phi_{s}\right\rangle_{H}\right) \mathrm{d} s\right)
\end{aligned}
$$


as $\epsilon \downarrow 0$.

We now prove

$$
\mathbb{E}\left(\int_{0}^{T} \mathrm{e}^{-2 \lambda_{0} s}\left\langle B\left(s, \tilde{Z}_{s}^{\epsilon}\right) \psi_{\epsilon}(s), \tilde{Z}_{s}^{\epsilon}\right\rangle_{H} \mathrm{~d} s\right) \rightarrow \mathbb{E}\left(\int_{0}^{T} \mathrm{e}^{-2 \lambda_{0} s}\left\langle B\left(s, \tilde{Z}_{s}\right) \psi(s), \tilde{Z}_{s}\right\rangle_{H} \mathrm{~d} s\right) .
$$

Since $\left\{\psi_{\epsilon}(\cdot, \omega)\right\}$ weakly converges to $\psi(\cdot, \omega)$ in $\bar{S}_{M} \quad$ (see (2)), then

$$
\begin{aligned}
& \lim _{\epsilon \downarrow 0} \mathbb{E}\left|\int_{0}^{T} \mathrm{e}^{-2 \lambda_{0} s}\left\langle B\left(s, \tilde{Z}_{s}\right)\left(\psi_{\epsilon}(s)-\psi(s)\right), \tilde{Z}_{s}\right\rangle_{H} \mathrm{~d} s\right| \\
& =\lim _{\epsilon \downarrow 0} \mathbb{E}\left|\int_{0}^{T}\left\langle\left(\psi_{\epsilon}(s)-\psi(s)\right), \mathrm{e}^{-2 \lambda_{0} s} B^{*}\left(s, \tilde{Z}_{s}\right) \tilde{Z}_{s}\right\rangle_{H} \mathrm{~d} s\right| \\
& =0,
\end{aligned}
$$

the last limit follows by using dominated convergence theorem. By (2), (H5), Lemma 1 and (19), we also have

$$
\begin{aligned}
& \mathbb{E}\left|\int_{0}^{T} \mathrm{e}^{-2 \lambda_{0} s}\left\langle B\left(s, \tilde{Z}_{s}^{\epsilon}\right) \psi_{\epsilon}(s), \tilde{Z}_{s}^{\epsilon}-\tilde{Z}_{s}\right\rangle_{H} \mathrm{~d} s\right| \\
& \leq c \mathbb{E}\left(\int_{0}^{T}\left(\left\|\tilde{Z}_{s}^{\epsilon}\right\|_{H}+1\right)\left\|\psi_{\epsilon}(s)\right\|_{G_{Q}}\left\|\tilde{Z}_{s}^{\epsilon}-\tilde{Z}_{s}\right\|_{H} \mathrm{~d} s\right) \\
& \leq c \mathbb{E}\left(\int_{0}^{T}\left(\left\|\tilde{Z}_{s}^{\epsilon}\right\|_{H}+1\right)^{2}\left\|\tilde{Z}_{s}^{\epsilon}-\tilde{Z}_{s}\right\|_{H}^{2} \mathrm{~d} s\right)^{1 / 2}\left(\int_{0}^{T}\left\|\psi_{\epsilon}(s)\right\|_{G_{Q}}^{2} \mathrm{~d} s\right)^{1 / 2} \\
& \leq c\left(\mathbb{E}\left(\sup _{s \in[0, T]}\left\|\tilde{Z}_{s}^{\epsilon}\right\|_{H}^{2}+1\right)\right)^{1 / 2}\left(\mathbb{E} \int_{0}^{T}\left\|\tilde{Z}_{s}^{\epsilon}-\tilde{Z}_{s}\right\|_{H}^{2} \mathrm{~d} s\right)^{1 / 2} \\
& \leq c\left(\mathbb{E} \int_{0}^{T}\left\|\tilde{Z}_{s}^{\epsilon}-\tilde{Z}_{s}\right\|_{H}^{2}\right)^{1 / 2} \rightarrow 0,
\end{aligned}
$$

and

$$
\begin{aligned}
& \mathbb{E}\left|\int_{0}^{T} \mathrm{e}^{-2 \lambda_{0} s}\left\langle\left(B\left(s, \tilde{Z}_{s}^{\epsilon}\right)-B\left(s, \tilde{Z}_{s}\right)\right) \psi_{\epsilon}(s), \tilde{Z}_{s}^{\epsilon}\right\rangle_{H} \mathrm{~d} s\right| \\
& \leq c \mathbb{E}\left(\int_{0}^{T}\left\|\tilde{Z}_{s}^{\epsilon}-\tilde{Z}_{s}\right\|_{H}\left\|\psi_{\epsilon}(s)\right\|_{G_{Q}}\left\|\tilde{Z}_{s}\right\|_{H} \mathrm{~d} s\right) \\
& \leq c \mathbb{E}\left(\int_{0}^{T}\left\|\tilde{Z}_{s}^{\epsilon}-\tilde{Z}_{s}\right\|_{H}^{2}\left\|\tilde{Z}_{s}\right\|_{H}^{2} \mathrm{~d} s\right)^{1 / 2} \\
& \leq c\left(\mathbb{E}\left(\sup _{s \in[0, T]}\left\|\tilde{Z}_{s}\right\|_{H}^{2}\right)\right)^{1 / 2}\left(\mathbb{E} \int_{0}^{T}\left\|\tilde{Z}_{s}^{\epsilon}-\tilde{Z}_{s}\right\|_{H}^{2} \mathrm{~d} s\right)^{1 / 2} \rightarrow 0 .
\end{aligned}
$$

Then limit (27) follows.

Moreover, it is easy to get that

$$
\lim _{\epsilon \downarrow 0} \epsilon \mathbb{E}\left(\int_{0}^{T} \mathrm{e}^{-2 \lambda_{0} s}\left\|B\left(s, \tilde{Z}_{s}^{\epsilon}\right)\right\|_{L_{Q}}^{2} \mathrm{~d} s\right)=0
$$

Now we prove the following limit:

$$
\begin{aligned}
& 2 \mathbb{E}\left(\int_{0}^{T} \int_{\mathbb{X}} \mathrm{e}^{-2 \lambda_{0} s}\left\langle\left(s, \tilde{Z}_{s}^{\epsilon}, x\right), \tilde{Z}_{s}^{\epsilon}\right\rangle_{H}\left(\varphi_{\epsilon}(s, x)-1\right) v(\mathrm{~d} x) \mathrm{d} s\right) \\
& \rightarrow 2 \mathbb{E}\left(\int_{0}^{T} \int_{\mathbb{X}} \mathrm{e}^{-2 \lambda_{0} s}\left\langle\left(s, \tilde{Z}_{s}, x\right), \tilde{Z}_{s}\right\rangle_{H}(\varphi(s, x)-1) v(\mathrm{~d} x) \mathrm{d} s\right)
\end{aligned}
$$

By (H5), Lemma 1 and (19), we have 


$$
\begin{aligned}
& \mathbb{E}\left|\int_{0}^{T} \int_{\mathbb{X}} \mathrm{e}^{-2 \lambda_{0} s}\left(\left\langle f\left(s, \tilde{Z}_{s}^{\epsilon}, x\right), \tilde{Z}_{s}^{\epsilon}\right\rangle_{H} \varphi_{\epsilon}(s, x)-\left\langle f\left(s, \tilde{Z}_{s}, x\right), \tilde{Z}_{s}\right\rangle_{H} \varphi(s, x)\right) v(\mathrm{~d} x) \mathrm{d} s\right| \\
& \leq \mathbb{E} \int_{0}^{T} \int_{\mathbb{X}}\left|\left\langle f\left(s, \tilde{Z}_{s}^{\epsilon}, x\right), \tilde{Z}_{s}^{\epsilon}-\tilde{Z}_{s}\right\rangle_{H} \varphi_{\epsilon}(s, x)\right| v(\mathrm{~d} x) \mathrm{d} s \\
& \quad+\mathbb{E} \int_{0}^{T} \int_{\mathbb{X}}\left|\left\langle f\left(s, \tilde{Z}_{s}^{\epsilon}, x\right)-f\left(s, \tilde{Z}_{s}, x\right), \tilde{Z}_{s}\right\rangle_{H} \varphi_{\epsilon}(s, x)\right| v(\mathrm{~d} x) \mathrm{d} s \\
& \quad+\mathbb{E}\left|\int_{0}^{T} \int_{\mathbb{X}} \mathrm{e}^{-2 \lambda_{0} s}\left\langle f\left(s, \tilde{Z}_{s}, x\right), \tilde{Z}_{s}\right\rangle_{H}\left(\varphi_{\epsilon}(s, x)-\varphi(s, x)\right) v(\mathrm{~d} x) \mathrm{d} s\right| \\
& \leq c \mathbb{E}\left(\int_{0}^{T} \int_{\mathbb{X}}\left(1+\left\|\tilde{Z}_{s}^{\epsilon}\right\|_{H}\right)\left\|\tilde{Z}_{s}^{\epsilon}-\tilde{Z}_{s}\right\|_{H} \varphi_{\epsilon}(s, x) v(\mathrm{~d} x) \mathrm{d} s\right) \\
& \quad+\mathbb{E}\left|\int_{0}^{T} \int_{\mathbb{X}} \mathrm{e}^{-2 \lambda_{0} s}\left\langle f\left(s, \tilde{Z}_{s}, x\right), \tilde{Z}_{s}\right\rangle_{H}\left(\varphi_{\epsilon}(s, x)-\varphi(s, x)\right) v(\mathrm{~d} x) \mathrm{d} s\right|=c J_{1}+J_{2},
\end{aligned}
$$

where

$$
J_{1}:=\mathbb{E}\left(\int_{0}^{T} \int_{\mathbb{X}}\left(1+\left\|\tilde{Z}_{s}^{\epsilon}\right\|_{H}\right)\left\|\tilde{Z}_{s}^{\epsilon}-\tilde{Z}_{s}\right\|_{H} \varphi_{\epsilon}(s, x) v(\mathrm{~d} x) \mathrm{d} s\right)
$$

and

$$
J_{2}:=\mathbb{E}\left|\int_{0}^{T} \int_{\mathbb{X}} \mathrm{e}^{-2 \lambda_{0} s}\left\langle f\left(s, \tilde{Z}_{s}, x\right), \tilde{Z}_{s}\right\rangle_{H}\left(\varphi_{\epsilon}(s, x)-\varphi(s, x)\right) v(\mathrm{~d} x) \mathrm{d} s\right| .
$$

For $J_{1}$, by Young inequality, we have

$$
\begin{aligned}
J_{1} \leq & \left(\mathbb{E} \int_{0}^{T} \int_{\mathbb{X}} \varphi_{\epsilon}(s, x) v(\mathrm{~d} x) \mathrm{d} s\right)^{1 / 2}\left\{\left(\mathbb{E} \int_{0}^{T} \int_{\mathbb{X}}\left\|\tilde{Z}_{s}^{\epsilon}-\tilde{Z}_{s}\right\|_{H}^{2} \varphi_{\epsilon}(s, x) v(\mathrm{~d} x) \mathrm{d} s\right)^{1 / 2}\right. \\
& \left.+\mathbb{E}\left(\int_{0}^{T} \int_{\mathbb{X}}\left\|\tilde{Z}_{s}^{\epsilon}-\tilde{Z}_{s}\right\|_{H}^{2}\left\|\tilde{Z}_{s}^{\epsilon}\right\|_{H}^{2} \varphi_{\epsilon}(s, x) v(\mathrm{~d} x) \mathrm{d} s\right)^{1 / 2}\right\} \\
\leq & c\left(\mathbb{E} \int_{0}^{T} \int_{\mathbb{X}}\left\|\tilde{Z}_{s}^{\epsilon}-\tilde{Z}_{s}\right\|_{H}^{2} \varphi_{\epsilon}(s, x) v(\mathrm{~d} x) \mathrm{d} s\right)^{1 / 2} \rightarrow 0, \quad \text { as } \epsilon \downarrow 0
\end{aligned}
$$

by noting (16) and (19). For $J_{2}$, by (4), (H6) and $\tilde{Z} \in C\left([0, T] ; V^{*}\right)$, it's easy to verify $\mathrm{e}^{-2 \lambda_{0} s}\left\langle f\left(s, \tilde{Z}_{s}, x\right), \tilde{Z}_{s}\right\rangle_{H}$ is a continuous function on $[0, T] \times \mathbb{X}$ with the compact support $[0, T] \times \Gamma$, and by the weak convergence of $v_{T}^{\varphi_{\epsilon}}$ to $v_{T}^{\varphi}$ (see Remark 1) and dominated convergence theorem, $J_{2} \rightarrow 0$ as $\epsilon \downarrow 0$. Then (30) goes to 0 as $\epsilon \downarrow 0$. Similarly, we have

$$
\mathbb{E}\left|\int_{0}^{T} \int_{\mathbb{X}} \mathrm{e}^{-2 \lambda_{0} s}\left(\left\langle f\left(s, \tilde{Z}_{s}^{\epsilon}, x\right), \tilde{Z}_{s}^{\epsilon}\right\rangle_{H}-\left\langle f\left(s, \tilde{Z}_{s}, x\right), \tilde{Z}_{s}\right\rangle_{H}\right) v(\mathrm{~d} x) \mathrm{d} s\right|, \quad \text { as } \epsilon \downarrow 0 .
$$

Then, we get (29).

It is obvious that

$$
\lim _{\epsilon \downarrow 0} \epsilon \mathbb{E} \int_{0}^{T} \int_{\mathbb{X}} \mathrm{e}^{-2 \lambda_{0} s}\left\|f\left(s, \tilde{Z}_{s}^{\epsilon}, x\right)\right\|_{H}^{2} \varphi_{\epsilon}(s, x) \mathrm{d} s v(\mathrm{~d} x) \rightarrow 0 .
$$

Combining (26) to (31) yields that

$$
\begin{aligned}
& \mathbb{E}\left(\mathrm{e}^{-2 \lambda_{0} T}\left\|\tilde{Z}_{T}\right\|_{H}^{2}\right) \leq \liminf _{\epsilon \downarrow} \mathbb{E}\left(\mathrm{e}^{-2 \lambda_{0} T}\left\|\tilde{Z}_{T}^{\epsilon}\right\|_{H}^{2}\right) \\
& \leq \\
& \qquad\|\|_{H}^{2}+2 \mathbb{E}\left(\int_{0}^{T} \mathrm{e}^{-2 \lambda_{0} s} V^{*}\left\langle Y_{s}-A\left(s, \Phi_{s}\right), \Phi_{s}\right\rangle_{V} \mathrm{~d} s\right)+2 \mathbb{E}\left(\int_{0}^{T} \mathrm{e}^{-2 \lambda_{0} s} V^{*}\left\langle A\left(s, \Phi_{s}\right), \tilde{Z}_{s}\right\rangle_{V} \mathrm{~d} s\right) \\
& +\lambda_{0} \mathbb{E}\left(\int_{0}^{T} \mathrm{e}^{-2 \lambda_{0} s}\left(\left\|\Phi_{s}\right\|_{H}^{2}-2\left\langle\tilde{Z}_{s}, \Phi_{s}\right\rangle_{H}\right) \mathrm{d} s\right)+2 \mathbb{E}\left(\int_{0}^{T} \mathrm{e}^{-2 \lambda_{0} s}\left\langle B\left(s, \tilde{Z}_{s}\right) \psi(s), \tilde{Z}_{s}\right\rangle_{H} \mathrm{~d} s\right) \\
& +2 \mathbb{E}\left(\int_{0}^{T} \int_{\mathbb{X}} \mathrm{e}^{-2 \lambda_{0} s}\left\langle f\left(s, \tilde{Z}_{s}, x\right), \tilde{Z}_{s}\right\rangle_{H}(\varphi(s, x)-1) v(\mathrm{~d} x) \mathrm{d} s\right) .
\end{aligned}
$$


On the other hand, by Itô's formula we have

$$
\begin{aligned}
\mathrm{e}^{-2 \lambda_{0} T}\left\|\hat{Z}_{T}\right\|_{H}^{2}= & \|z\|_{H}^{2}-2 \lambda_{0} \int_{0}^{T} \mathrm{e}^{-2 \lambda_{0} s}\left\|\tilde{Z}_{s}\right\|_{H}^{2} \mathrm{~d} s+2 \int_{0}^{T} \mathrm{e}^{-2 \lambda_{0} s} V^{*}\left\langle Y_{s}, \tilde{Z}_{s}\right\rangle_{V} \mathrm{~d} s \\
& +2 \int_{0}^{T} \mathrm{e}^{-2 \lambda_{0} s}\left\langle B\left(s, \tilde{Z}_{s}\right) \psi(s), \tilde{Z}_{s}\right\rangle_{H} \mathrm{~d} s \\
& +2 \int_{0}^{T} \int_{\mathbb{X}} \mathrm{e}^{-2 \lambda_{0} s}\left\langle f\left(s, \tilde{Z}_{s}, x\right), \tilde{Z}_{s}\right\rangle_{H}(\varphi(s, x)-1) v(\mathrm{~d} x) \mathrm{d} s
\end{aligned}
$$

So, we have

$$
\mathbb{E}\left(\int_{0}^{T} \mathrm{e}^{-2 \lambda_{0} s}{ }_{V^{*}}\left\langle Y_{s}-A\left(s, \Phi_{s}\right), \tilde{Z}_{s}-\Phi_{s}\right\rangle_{V} \mathrm{~d} s\right) \leq \lambda_{0} \mathbb{E}\left(\int_{0}^{T} \mathrm{e}^{-2 \lambda_{0} s}\left\|\tilde{Z}_{s}-\Phi_{s}\right\|_{H}^{2} \mathrm{~d} s\right),
$$

which implies (24) by (H1).

Step 3. In this step we prove (13) and (14). Notice that

$$
\begin{aligned}
\tilde{Z}_{t}^{\epsilon}-\tilde{Z}_{t}= & \int_{0}^{t}\left(A\left(s, \tilde{Z}_{s}^{\epsilon}\right)-A\left(s, \tilde{Z}_{s}\right)\right) \mathrm{d} s+\int_{0}^{t}\left(B\left(s, \tilde{Z}_{s}^{\epsilon}\right) \psi_{\epsilon}(s)-B\left(s, \tilde{Z}_{s}\right) \psi(s)\right) \mathrm{d} s \\
& +\int_{0}^{t} \int_{\mathbb{X}}\left(f\left(s, \tilde{Z}_{s-}^{\epsilon}, x\right) \varphi_{\epsilon}(s, x)-f\left(s, \tilde{Z}_{s}, x\right) \varphi(s, x)\right) v(\mathrm{~d} x) \mathrm{d} s \\
& -\int_{0}^{t} \int_{\mathbb{X}}\left(f\left(s, \tilde{Z}_{s-}^{\epsilon}, x\right)-f\left(s, \tilde{Z}_{s-}, x\right)\right) v(\mathrm{~d} x) \mathrm{d} s+\sqrt{\epsilon} \int_{0}^{t} B\left(s, \tilde{Z}_{s}^{\epsilon}\right) \mathrm{d} W_{s} \\
& +\epsilon \int_{0}^{t} \int_{\mathbb{X}} f\left(s, \tilde{Z}_{s-}^{\epsilon}, x\right)\left(N^{\epsilon^{-1} \varphi_{\epsilon}}(\mathrm{d} s, \mathrm{~d} x)-\epsilon^{-1} \varphi_{\epsilon}(s, x) v(\mathrm{~d} x) \mathrm{d} s\right) .
\end{aligned}
$$

By Itô's formula, we have

$$
\left\|\tilde{Z}_{t}^{\epsilon}-\tilde{Z}_{t}\right\|_{H}^{2}=\sum_{i=0}^{9} I_{i}^{\epsilon}(t)
$$

where

$$
\begin{gathered}
I_{0}^{\epsilon}(t):=2 \int_{0}^{t} v^{*}\left\langle A\left(s, \tilde{Z}_{s}^{\epsilon}\right)-A\left(s, \tilde{Z}_{s}\right), \tilde{Z}_{s}^{\epsilon}-\tilde{Z}_{s}\right\rangle_{V} \mathrm{~d} s, \\
I_{1}^{\epsilon}(t):=2 \int_{0}^{t}\left\langle B\left(s, \tilde{Z}_{s}^{\epsilon}\right) \psi_{\epsilon}(s), \tilde{Z}_{s}^{\epsilon}-\tilde{Z}_{s}\right\rangle_{H} \mathrm{~d} s, \\
I_{2}^{\epsilon}(t):=-2 \int_{0}^{t}\left\langle B\left(s, \tilde{Z}_{s}\right) \psi(s), \tilde{Z}_{s}^{\epsilon}-\tilde{Z}_{s}\right\rangle_{H} \mathrm{~d} s, \\
I_{3}^{\epsilon}(t):=2 \int_{0}^{t} \int_{\mathbb{X}}\left\langle f\left(s, \tilde{Z}_{s-}^{\epsilon}, x\right) \varphi_{\epsilon}(s, x), \tilde{Z}_{s}^{\epsilon}-\tilde{Z}_{s}\right\rangle_{H} v(\mathrm{~d} x) \mathrm{d} s, \\
I_{4}^{\epsilon}(t):=-2 \int_{0}^{t} \int_{\mathbb{X}}\left\langle f\left(s, \tilde{Z}_{s}, x\right) \varphi(s, x), \tilde{Z}_{s}^{\epsilon}-\tilde{Z}_{s}\right\rangle_{H} v(\mathrm{~d} x) \mathrm{d} s, \\
I_{5}^{\epsilon}(t):=-2 \int_{0}^{t} \int_{\mathbb{X}}\left\langle f\left(s, \tilde{Z}_{s-}^{\epsilon}, x\right)-f\left(s, \tilde{Z}_{s-}, x\right), \tilde{Z}_{s}^{\epsilon}-\tilde{Z}_{s}\right\rangle_{H} v(\mathrm{~d} x) \mathrm{d} s, \\
I_{6}^{\epsilon}(t):=2 \sqrt{\epsilon} \int_{0}^{t}\left\langle B\left(s, \tilde{Z}_{s}^{\epsilon}\right) \mathrm{d} W_{s}, \tilde{Z}_{s}^{\epsilon}-\tilde{Z}_{s}\right\rangle_{H}, \\
I_{7}^{\epsilon}(t):=\epsilon \int_{0}^{t}\left\|B\left(s, \tilde{Z}_{s}^{\epsilon}\right)\right\|_{L_{Q}} \mathrm{~d} s, \\
I_{8}^{\epsilon}(t):=\int_{0}^{t} \int_{\mathbb{X}}\left(\left\|f\left(s, \tilde{Z}_{s-}^{\epsilon}, x\right)\right\|_{H}^{2}+2\left\langle\tilde{Z}_{s-}^{\epsilon}, f\left(s, \tilde{Z}_{s-}^{\epsilon}, x\right)\right\rangle_{H}\right) \\
\times\left(\epsilon N^{\epsilon^{-1} \varphi_{\epsilon}}(\mathrm{d} t, \mathrm{~d} x)-\varphi_{\epsilon}(s, x) v(\mathrm{~d} x) \mathrm{d} s\right), \\
I_{9}^{\epsilon}(t):=\epsilon \int_{0}^{t} \int_{\mathbb{X}}\left\|f\left(s, \tilde{Z}_{s}^{\epsilon}, x\right)\right\|_{H}^{2} \varphi_{\epsilon}(s, x) v(\mathrm{~d} x) \mathrm{d} s .
\end{gathered}
$$


By Lemma 1 and BDG's inequality, we get

$$
\lim _{\epsilon \rightarrow 0} \mathbb{E}\left(\sup _{t \in[0, T]} \sum_{i=6}^{9}\left|I_{i}^{\epsilon}(t)\right|\right) \rightarrow 0 .
$$

For $I_{i}^{\epsilon}$, we have

$$
\begin{aligned}
\mathbb{E}\left(\sup _{t \in[0, T]}\left|I_{1}^{\epsilon}(t)\right|\right) & \leq c \mathbb{E}\left(\int_{0}^{t}\left(\left\|\tilde{Z}_{s}^{\epsilon}\right\|_{H}+1\right)\left\|\psi_{\epsilon}(s)\right\|_{G_{Q}}\left\|\tilde{Z}_{s}^{\epsilon}-\tilde{Z}_{s}\right\|_{H} \mathrm{~d} s\right) \\
& \leq c \mathbb{E}\left(\int_{0}^{T}\left(\left\|\tilde{Z}_{s}^{\epsilon}\right\|_{H}+1\right)^{2}\left\|\tilde{Z}_{s}^{\epsilon}-\tilde{Z}_{s}\right\|_{H}^{2} \mathrm{~d} s\right)^{1 / 2} \\
& \leq c \mathbb{E}\left(\sup _{t \in[0, T]}\left\|\tilde{Z}_{s}^{\epsilon}\right\|_{H}^{2} \int_{0}^{T}\left\|\tilde{Z}_{s}^{\epsilon}-\tilde{Z}_{s}\right\|_{H}^{2} \mathrm{~d} s\right)^{1 / 2} \\
& \leq c\left(\mathbb{E} \sup _{t \in[0, T]}\left\|\tilde{Z}_{s}^{\epsilon}\right\|_{H}^{2}\right)^{1 / 2}\left(\mathbb{E} \int_{0}^{T}\left\|\tilde{Z}_{s}^{\epsilon}-\tilde{Z}_{s}\right\|_{H}^{2} \mathrm{~d} s\right)^{1 / 2} \\
& \leq c\left(\mathbb{E} \int_{0}^{T}\left\|\tilde{Z}_{s}^{\epsilon}-\tilde{Z}_{s}\right\|_{H}^{2} \mathrm{~d} s\right)^{1 / 2} \rightarrow 0, \text { as } \epsilon \downarrow 0 .
\end{aligned}
$$

Similarly

$$
\lim _{\epsilon \downarrow 0} \mathbb{E}\left(\sup _{t \in[0, T]}\left|I_{2}^{\epsilon}(t)\right|\right)=0 .
$$

For $I_{3}^{\epsilon}$, like $J_{1}$, we have

$$
\begin{aligned}
\mathbb{E}\left(\sup _{t \in[0, T]}\left|I_{3}^{\epsilon}(t)\right|\right) & \leq 2 \mathbb{E}\left(\int_{0}^{t} \int_{\mathbb{X}}\left\|f\left(s, \tilde{Z}_{s-}^{\epsilon}, x\right)\right\|_{H}\left\|\tilde{Z}_{s}^{\epsilon}-\tilde{Z}_{s}\right\|_{H} \varphi_{\epsilon}(s, x) v(\mathrm{~d} x) \mathrm{d} s\right) \\
& \leq c\left(\mathbb{E} \int_{0}^{T} \int_{\mathbb{X}}\left\|\tilde{Z}_{s}^{\epsilon}-\tilde{Z}_{s}\right\|_{H}^{2} \varphi_{\epsilon}(s, x) v(\mathrm{~d} x) \mathrm{d} s\right)^{1 / 2} \\
& \leq c_{M}\left(\mathbb{E} \int_{0}^{T}\left\|\tilde{Z}_{s}^{\epsilon}-\tilde{Z}_{s}\right\|_{H}^{2} \mathrm{~d} s\right)^{1 / 2} \rightarrow 0, \text { as } \epsilon \downarrow 0 .
\end{aligned}
$$

Similarly

$$
\lim _{\epsilon \downarrow 0} \mathbb{E}\left(\sup _{t \in[0, T]}\left|I_{4}^{\epsilon}(t)\right|\right)=0 .
$$

For $I_{5}^{\epsilon}$, by (H5) and (H6) we have

$$
\begin{aligned}
\mathbb{E}\left(\sup _{t \in[0, T]}\left|I_{5}^{\epsilon}(t)\right|\right) & \leq 2 \mathbb{E} \int_{0}^{t} \int_{\mathbb{X}}\left\|f\left(s, \tilde{Z}_{s-}^{\epsilon}, x\right)-f\left(s, \tilde{Z}_{s-}, x\right)\right\|_{H}\left\|\tilde{Z}_{s}^{\epsilon}-\tilde{Z}_{s}\right\|_{H} v(\mathrm{~d} x) \mathrm{d} s \\
& \leq 2 c v(\Gamma) \mathbb{E}\left(\int_{0}^{T}\left\|\tilde{Z}_{s}^{\epsilon}-\tilde{Z}_{s}\right\|_{H}^{2} \mathrm{~d} s\right) \rightarrow 0 \text {, as } \epsilon \downarrow 0 .
\end{aligned}
$$

Assume $\lambda_{1}>0$, then

$$
I_{0}^{\epsilon}(t) \leq-\lambda_{1} \int_{0}^{t}\left\|\tilde{Z}_{s}^{\epsilon}-\tilde{Z}_{s}\right\|_{V}^{2} \mathrm{~d} s+\lambda_{0} \int_{0}^{t}\left\|\tilde{Z}_{s}^{\epsilon}-\tilde{Z}_{s}\right\|_{H}^{2} \mathrm{~d} s
$$

Set

$$
F(t):=\limsup _{\epsilon \downarrow 0} \mathbb{E}\left(\sup _{s \in[0, t]}\left\|\tilde{Z}_{s}^{\epsilon}-\tilde{Z}_{s}\right\|_{H}^{2}\right),
$$

then 


$$
F(t) \leq \lambda_{0} \int_{0}^{t} F(s) \mathrm{d} s=0 .
$$

So

$$
F(T)=0 .
$$

Notice (32), we get (13) and (14) immediately.

We also have the following main lemma.

Lemma 4. There exists a probability space $(\bar{\Omega}, \overline{\mathcal{F}}, \bar{P})$ and a sequence (for convenience, still denote by $\epsilon)\left\{\left(\bar{\psi}_{\epsilon}, \bar{\varphi}_{\epsilon}\right), \bar{Z}^{\epsilon},\left(\sqrt{\epsilon} \bar{W}+\int_{0} \bar{\psi}_{\epsilon}(s) \mathrm{d} s, \epsilon \bar{N}^{\epsilon^{-1} \bar{\varphi}_{\epsilon}}\right)\right\}$ and

$\left\{(\bar{\psi}, \bar{\varphi}), \bar{Z},\left(\int_{0} \bar{\psi}(s) \mathrm{d} s, v_{T}^{\bar{\varphi}}\right)\right\}$ defined on this space and taking value in $\mathcal{U}_{M} \times D\left([0, T] ; V^{*}\right) \times \Omega$ with $\bar{Z} \in C\left([0, T] ; V^{*}\right)$ such that:

1) For each $\epsilon,\left\{\left(\bar{\psi}_{\epsilon}, \bar{\varphi}_{\epsilon}\right), \bar{Z}^{\epsilon},\left(\sqrt{\epsilon} \bar{W}+\int_{0} \bar{\psi}_{\epsilon}(s) \mathrm{d} s, \epsilon \bar{N}^{\epsilon^{-1} \bar{\varphi}_{\epsilon}}\right)\right\}$ has the same law as $\left\{\left(\psi_{\epsilon}, \varphi_{\epsilon}\right), \tilde{Z}^{\epsilon},\left(\sqrt{\epsilon} \bar{W}+\int_{0} \psi_{\epsilon}(s) \mathrm{d} s, \epsilon N^{\epsilon^{-1} \varphi_{\epsilon}}\right)\right\} ;$

2) $\left\{\left(\bar{\psi}_{\epsilon}, \bar{\varphi}_{\epsilon}\right), \bar{Z}^{\epsilon}\right\} \rightarrow\{(\bar{\psi}, \bar{\varphi}), \bar{Z}\}$ in $\mathcal{U}_{M} \times D\left([0, T] ; V^{*}\right) \times \Omega, \bar{P}$-a.s., as $\epsilon \rightarrow 0$;

3) $\{(\bar{\psi}, \bar{\varphi}), \bar{Z}\}$ uniquely solves the following equation:

$$
\begin{aligned}
\bar{Z}_{t}= & z+\int_{0}^{t} A\left(s, \bar{Z}_{s}\right) \mathrm{d} s+\int_{0}^{t} B\left(s, \bar{Z}_{s}\right) \bar{\psi}(s) \mathrm{d} s \\
& +\int_{0}^{t} \int_{\mathbb{X}} f\left(s, \bar{Z}_{s}, x\right)(\bar{\varphi}(s, x)-1) v(\mathrm{~d} x) \mathrm{d} s
\end{aligned}
$$

Moreover, we have

$$
\mathbb{E}^{\bar{P}}\left(\sup _{0 \leq t \leq T}\left\|\bar{Z}_{t}^{\epsilon}-\bar{Z}_{t}\right\|_{H}^{2}\right) \rightarrow 0
$$

and if $\lambda_{1}>0$, then

$$
\mathbb{E}^{\bar{P}} \int_{0}^{T}\left\|\bar{Z}_{t}^{\epsilon}-\bar{Z}_{t}\right\|_{V}^{2} \mathrm{~d} t \rightarrow 0
$$

Proof. From Corollary 1, we have $\left\{\tilde{Z}^{\epsilon}\right\}$ is $C$-tight in $D\left([0, T] ; V^{*}\right)$. By the compactness of $S_{M}$, the law of $\left\{\left(\psi_{\epsilon}, \varphi_{\epsilon}\right), \tilde{Z}^{\epsilon}\right\}$ in $S_{M} \times D\left([0, T] ; V^{*}\right)$ is tight. By Skorokhod's embedding theorem, (1) and (2) hold. Since $\bar{Z}_{0}^{\epsilon}=z \quad \bar{P}$-a.s. and

$$
\begin{aligned}
\bar{Z}_{t}^{\epsilon}= & z+\int_{0}^{t} A\left(s, \bar{Z}_{s}^{\epsilon}\right) \mathrm{d} s+\int_{0}^{t} B\left(s, \bar{Z}_{s}^{\epsilon}\right) \bar{\psi}_{\epsilon}(s) \mathrm{d} s+\sqrt{\epsilon} \int_{0}^{t} B\left(s, \bar{Z}_{s}^{\epsilon}\right) \mathrm{d} \bar{W}_{s} \\
& +\int_{0}^{t} \int_{\mathbb{X}} f\left(s, \bar{Z}_{s-}^{\epsilon}, x\right)\left(\bar{\varphi}_{\epsilon}(s, x)-1\right) \mathrm{d} s v(\mathrm{~d} x) \\
& +\epsilon \int_{0}^{t} \int_{\mathbb{X}} f\left(s, \bar{Z}_{s-}^{\epsilon}, x\right)\left(\bar{N}^{\epsilon^{-1} \bar{\varphi}_{\epsilon}}(\mathrm{d} s, \mathrm{~d} x)-\epsilon^{-1} \bar{\varphi}_{\epsilon}(s, x) \mathrm{d} s v(\mathrm{~d} x)\right) .
\end{aligned}
$$

Then, the other conclusions follow from Lemma 3 and noting for $\bar{P}$ almost all $\omega$, $\bar{Z}(\omega) \in C\left([0, T] ; V^{*}\right)$.

Remark 5. Assume that (H1)-(H7) and $\lambda_{1}>0$ hold, we have verified Hypothesis (1) by the above lemma.

For fixed $M \in \mathbb{N}$, let $(h, g) \in S_{M}$ and let $\mathcal{G}^{0}: \Omega \rightarrow \mathbb{D}$ such that $\mathcal{G}^{0}\left(\int_{0} h(s) \mathrm{d} s, v_{T}^{g}\right)$ is the unique solution of

$$
Z_{t}=z+\int_{0}^{t} A\left(s, Z_{s}\right) \mathrm{d} s+\int_{0}^{t} B\left(s, Z_{s}\right) h(s) \mathrm{d} s+\int_{0}^{t} \int_{\mathbb{X}} f\left(s, Z_{s}, x\right)(g(s, x)-1) \mathrm{d} s v(\mathrm{~d} x) .
$$


We point out that the difference between $(h, g)$ in the above equation and $\left(\psi_{\epsilon}, \varphi_{\epsilon}\right)$ in (13) is that $(h, g)$ is not random. We have the following result.

Lemma 5. Assume that (H1)-(H7) and $\lambda_{1}>0$ hold. Let $\left(h_{m}, g_{m}\right),(h, g) \in S_{M}$ be such that $\left(h_{m}, g_{m}\right) \rightarrow(h, g)$ in the weak topology of $S_{M}$ (see Section 2$)$, then

$$
\mathcal{G}^{0}\left(\int_{0} h_{m}(s) \mathrm{d} s, v_{T}^{g_{m}}\right) \rightarrow \mathcal{G}^{0}\left(\int_{0} h(s) \mathrm{d} s, v_{T}^{g}\right) .
$$

Proof. Similar to the proofs of Lemma 1 and 2, we can get $\left\{\mathcal{G}^{0}\left(\int_{0} h_{m}(s) \mathrm{d} s, v_{T}^{g_{m}}\right)\right\}_{M \in \mathbb{N}}$ is $C$-tight. As in Lemma 4, there exist a subsequence $m_{k}$ (still denoted by $m$ ) and $Z^{0} \in C\left([0, T] ; V^{*}\right)$ satisfying

$$
\sup _{t \in[0, T]}\left\|\mathcal{G}^{0}\left(\int_{0} h_{m}(s) \mathrm{d} s, v_{T}^{g_{m}}\right)-Z^{0}\right\|_{V^{*}} \rightarrow 0, \quad \text { as } m \rightarrow \infty .
$$

Combining with this convergence and the method used in the proof of Lemma 3, we have $\mathcal{G}^{0}\left(\int_{0} h(s) \mathrm{d} s, v_{T}^{g}\right)=Z^{0}$, then the result holds.

Using Remark 5, Lemma 5 and Theorem 2, we obtain the following large deviation principle.

Theorem 6. Under the same assumptions as in Lemma $5,\left\{Z^{\epsilon}\right\}_{\epsilon>0}$ satisfies a large deviation principle with rate function I defined as in (3), i.e. for any $A \in \mathcal{B}(\mathbb{D})$

$$
-\inf _{\phi \in A^{\circ}} I(\phi) \leq \liminf _{\epsilon \rightarrow 0} \epsilon \log \mu_{\epsilon}(A) \leq \limsup \epsilon \log \mu_{\epsilon}(A) \leq-\inf _{\phi \in A} I(\phi),
$$

where $\mu_{\epsilon}$ is the law of $Z^{\epsilon}$ in $\mathbb{D}$ and $\mathbb{D}$ is $D([0, T] ; H) \cap L^{2}([0, T] ; V)$.

Remark 7. If $\lambda_{1}=0$, then the conclusion still holds if $\mathbb{D}$ is replaced by $D([0, T] ; H)$.

\section{Application-Stochastic Porous Medium Equation}

Similar to [9], consider a bounded domain $\mathcal{O}$ in $\mathbb{R}^{d}$ with smooth boundary. For $p \geq 2$, let

$$
V:=L^{p}(\mathcal{O}), H:=W^{-1,2}(\mathcal{O}), V^{*}:=L^{p /(p-1)}(\mathcal{O}) .
$$

The inner product in $H$ is defined by

$$
\langle x, y\rangle_{H}:=\int_{\mathcal{O}}(-\Delta)^{-1 / 2} x(s) \cdot(-\Delta)^{-1 / 2} y(s) \mathrm{d} s, \quad x, y \in W^{-1,2}(\mathcal{O}) .
$$

$-\Delta$ establish an isomorphism between $W^{-1,2}(\mathcal{O})$ and $W_{0}^{1,2}(\mathcal{O})$. We identify $W_{0}^{1,2}(\mathcal{O})$ with the dual space $H^{*}$ and $H$, then $H^{*}=W_{0}^{1,2}(\mathcal{O}) \subset L^{p /(p-1)}(\mathcal{O})$. Therefore

$$
V \subset H \equiv H^{*} \subset V^{*},
$$

and the inclusions are compact.

Let $\phi_{p}(r):=r|r|^{p-2}$. For $x \in V$, denote by

$$
A(x):=\Delta \phi_{p}(x) .
$$

Then $A(x) \in V^{*}$ and (H1)-(H4) hold (cf. [9] [16]).

Let $B_{1}, \cdots, B_{n} \in L_{2}\left(Q^{1 / 2} G, H\right)$. Define 


$$
B(t, x):=\sum_{k=1}^{n} g_{k}\left(\left(e_{n_{1}}, x\right)_{H}, \cdots,\left(e_{n_{k}}, x\right)_{H}\right) B_{k}, e_{n_{j}} \in H,
$$

where $g_{k}$ are Lipschitz continuous on $R^{n_{k}}$. Let $\mathbb{X}:=\mathbb{R}, h_{1}, \cdots, h_{n} \in H$, and define

$$
f(t, x, y):=\sum_{k=1}^{n} f_{k}\left(\left(e_{n_{1}}, x\right)_{H}, \cdots,\left(e_{n_{k}}, x\right)_{H}\right) h_{k} 1_{[0,6]}(y), e_{n_{j}} \in H,
$$

where $f_{k}$ are Lipschitz continuous on $R^{n_{k}}$. Then $B$ and $f$ satisfy (H5)-(H6).

Consider the following stochastic porous medium equation

$$
\left\{\begin{array}{l}
\mathrm{d} Z_{t}^{\epsilon}=A\left(Z_{t}^{\epsilon}\right) \mathrm{d} t+\sqrt{\epsilon} B\left(t, Z_{t}^{\epsilon}\right) \mathrm{d} W_{t}+\int_{\mathbb{X}} f\left(t, Z_{t-}^{\epsilon}, x\right)\left(\epsilon N^{\epsilon^{-1}}(\mathrm{~d} x, \mathrm{~d} t)-v(\mathrm{~d} x) \mathrm{d} t\right), \epsilon \in(0,1], \\
Z^{\epsilon}(t, \xi)=0, \forall \xi \in \partial \mathcal{O}, \\
Z^{\epsilon}(0, \xi)=Z(\xi) \in H .
\end{array}\right.
$$

Let $v_{\epsilon}$ be the law of $Z^{\epsilon}$ in $D([0, T] ; H) \cap L^{p}(0, T ; V)$. Then the conclusion of Theorem 6 holds.

\section{Acknowledgements}

The authors thank the Editor and the referee for their valuable comments. This work is supported in part by Zhejiang Provincial Natural Science Foundation of China (Grant No. LQ13A010020) and the National Natural Science Foundation of China (Grant No. 11401029).

\section{References}

[1] Dupuis, P. and Ellis, R.S. (1997) A Weak Convergence Approach to the Theory of Large Deviations. Wiley Series in Probability and Statistics: Probability and Statistics. A WileyInterscience Publication. John Wiley and Sons, Inc., New York. http://dx.doi.org/10.1002/9781118165904

[2] Boué, M. and Dupuis, P. (1998) A Variational Representation for Certain Functionals of Brownian Motion. Annals of Probability, 26, 1641-1659.

[3] Zhang, X. (2009) A Variational Representation for Random Funcionals on Abstract Wiener Spaces. Journal of Mathematics of Kyoto University, 49, 475-490.

[4] Zhang, X. (2009) Clark-Ocone Formula and Variational Representation for Poisson Functionals. Annals of Probability, 37, 506-529. http://dx.doi.org/10.1214/08-AOP411

[5] Budhiraja, A., Dupuis, P. and Maroulas, V. (2011) Variational Representations for Continuous Time Process. Annales de 1 Institut Henri Poincaré Probabilités et Statistiques, 47, 725-747.

[6] Boué, M., Dupuis, P. and Ellis, R.S. (2000) Large Deviations for Small Noise Diffusions with Discontinuous Statistics. Probability Theory and Related Fields, 116, 125-149. http://dx.doi.org/10.1007/PL00008720

[7] Budhiraja, A., Dupuis, P. and Maroulas, V. (2008) Large Deviations for Infinite Dimensional Stochastic Dynamical Systems. Annals of Probability, 36, 1390-1420. http://dx.doi.org/10.1214/07-AOP362

[8] Liu, W. (2010) Large Deviation for Stochastic Evolution Equations with Small Multiplicative Noise. Applied Mathematics \& Optimization, 61, 27-56. http://dx.doi.org/10.1007/s00245-009-9072-2 
[9] Ren, J. and Zhang, X. (2008) Freidlin-Wentzell's Large Deviations for Stochastic Evolution Equations. Journal of Functional Analysis, 254, 3148-3172.

http://dx.doi.org/10.1016/j.jfa.2008.02.010

[10] Zhang, X. (2008) Euler Schemes and Large Deviations for Stochastic Volterra Equations with Singular Kernels. Journal of Differential Equations, 224, 2226-2250.

http://dx.doi.org/10.1016/j.jde.2008.02.019

[11] Röckner, M. and Zhang, T. (2007) Stochastic Evolution Equations of Jump Type: Existence, Uniqueness and Large Deviation Principles. Potential Analysis, 26, 255-279. http://dx.doi.org/10.1007/s11118-006-9035-Z

[12] Budhiraja, A., Chen, J. and Dupuis, P. (2013) Large Deviations for Stochastic Partial Differential Equations Driven by a Poisson Random Measure. Stochastic Processes and their Applications, 123, 523-560. http://dx.doi.org/10.1016/j.spa.2012.09.010

[13] Gyöngy, I. and Krylov, N.V. (1980) On Stochastic Equations with Respect to Semimartingales I. Stochastics, 4, 1-21. http://dx.doi.org/10.1080/03610918008833154

[14] Gyöngy, I. and Krylov, N.V. (1982) On Stochastic Equations with Respect to Semimartingales II, Ito Formula in Banach Spaces. Stochastics, 6, 153-174. http://dx.doi.org/10.1080/17442508208833202

[15] Gyöngy, I. (1982) On Stochastic Equations with Respect to Semimartingales III. Stochastics, 7, 231-254. http://dx.doi.org/10.1080/17442508208833220

[16] Ren, J., Röckner, M. and Wang, F. (2007) Stochastic Generalixed Porous Media and fast Diffusion Equation. Journal of Differential Equations, 238, 118-152.

http://dx.doi.org/10.1016/j.jde.2007.03.027

[17] Zhao, H. (2009) On Existence and Uniqueness of Stochastic Evolution Equation with Poisson Jumps. Statistics \& Probability Letters, 79, 2367-2373. http://dx.doi.org/10.1016/j.spl.2009.08.006

[18] Yang, X., Zhai, J. and Zhang, T. (2015) Large Deviations for SPDEs of Jump Type. Stochastic and Dynamics, 15.

[19] Dadashi, H. (2013) Large Deviation Principle for Mild Solutions of Stochastic Evolution Equations with Multiplicative Lévy Noise. arXiv:1309.1935v1 [math.PR] http://arxiv.org/pdf/1309.1935.pdf

[20] Jacod, J. and Shiryaev, A. (1980) Limit Theorems for Stochastic Processes. Springer-Verlag, New York.

[21] Kallenberg, O. (2002) Foudations of Modern Probability. 2nd Edition, Applied Probability Trust. http://dx.doi.org/10.1007/978-1-4757-4015-8 
Submit or recommend next manuscript to SCIRP and we will provide best service for you:

Accepting pre-submission inquiries through Email, Facebook, LinkedIn, Twitter, etc. A wide selection of journals (inclusive of 9 subjects, more than 200 journals)

Providing 24-hour high-quality service

User-friendly online submission system

Fair and swift peer-review system

Efficient typesetting and proofreading procedure

Display of the result of downloads and visits, as well as the number of cited articles

Maximum dissemination of your research work

Submit your manuscript at: http://papersubmission.scirp.org/

Or contactapm@scirp.org 